\title{
SEARCHES FOR EXTENDED AND POINT-LIKE NEUTRINO SOURCES WITH FOUR YEARS OF ICECUBE DATA
}

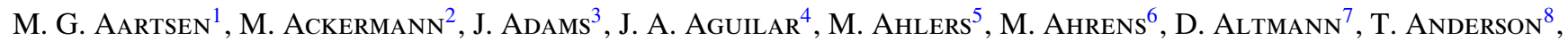

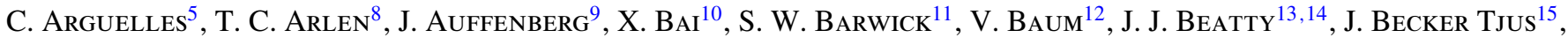
K.-H. BeCKer ${ }^{16}$, S. BenZvi ${ }^{5}$, P. Berghaus ${ }^{2}$, D. Berley ${ }^{17}$, E. Bernardini ${ }^{2}$, A. Bernhard ${ }^{18}$, D. Z. Besson ${ }^{19}$, G. Binder ${ }^{20,21}$,

D. Bindig ${ }^{16}$, M. BissoK ${ }^{9}$, E. Blaufuss ${ }^{17}$, J. Blumenthal ${ }^{9}$, D. J. Boersma ${ }^{22}$, C. Bohm ${ }^{6}$, F. Bos ${ }^{15}$, D. Bose ${ }^{23}$, S. Böser ${ }^{24}$, O. Botner ${ }^{22}$, L. Brayeur ${ }^{25}$, H.-P. Bretz ${ }^{2}$, A. M. Brown ${ }^{3}$, J. Casey ${ }^{26}$, M. Casier $^{25}$, E. Cheung ${ }^{17}$, D. Chirkin ${ }^{5}$, A. Christov ${ }^{4}$, B. Christy ${ }^{17}$, K. Clark ${ }^{27}$, L. Classen ${ }^{7}$, F. ClevermanN ${ }^{28}$, S. Coenders ${ }^{18}$, D. F. Cowen ${ }^{8,29}$, A. H. Cruz Silva ${ }^{2}$,

M. Danninger ${ }^{6}$, J. Daughhetee ${ }^{26}$, J. C. Davis ${ }^{13}$, M. DaY ${ }^{5}$, J. P. A. M. De ANDrÉ ${ }^{8}$, C. De ClercQ ${ }^{25}$, S. De Ridder ${ }^{30}$, P. Desiati ${ }^{5}$, K. D. de Vries ${ }^{25}$, M. De With ${ }^{31}$, T. DeYoung ${ }^{8}$, J. C. Díaz-Vélez ${ }^{5}$, M. DunKman ${ }^{8}$, R. Eagan ${ }^{8}$, B. Eberhardt ${ }^{12}$,

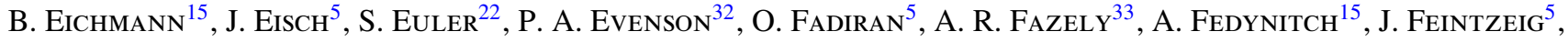
J. Felde $^{17}$, T. Feusels ${ }^{30}$, K. Filimonov ${ }^{21}$, C. Finley ${ }^{6}$, T. Fischer-Wasels ${ }^{16}$, S. Flis ${ }^{6}$, A. FrancKowiak ${ }^{24}$, K. Frantzen ${ }^{28}$,

T. Fuchs $^{28}$, T. K. Gaisser ${ }^{32}$, J. Gallagher ${ }^{34}$, L. GerhardT ${ }^{20,21}$, D. Gier ${ }^{9}$, L. Gladstone ${ }^{5}$, T. GlüsenKamp ${ }^{2}$,

A. GoldschmidT ${ }^{20}$, G. Golup ${ }^{25}$, J. G. Gonzalez ${ }^{32}$, J. A. Goodman ${ }^{17}$, D. Góra ${ }^{2}$, D. T. Grandmont ${ }^{35}$, D. Grant ${ }^{35}$, P. Gretskov ${ }^{9}$, J. C. Groh ${ }^{8}$, A. Groß ${ }^{18}$, C. HA ${ }^{20,21}$, C. HAACK $^{9}$, A. HaJ Ismail ${ }^{30}$, P. Hallen ${ }^{9}$, A. Hallgren ${ }^{22}$, F. Halzen ${ }^{5}$, K. Hanson ${ }^{36}$, D. Hebecker ${ }^{24}$, D. Heereman ${ }^{36}$, D. Heinen ${ }^{9}$, K. Helbing ${ }^{16}$, R. Hellauer ${ }^{17}$, D. Hellwig ${ }^{9}$, S. HicKford ${ }^{3}$,

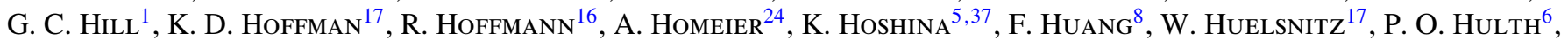

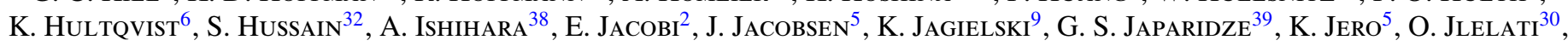

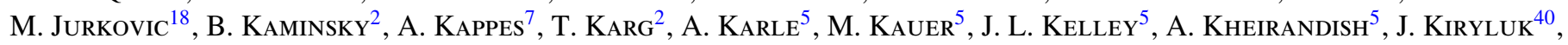

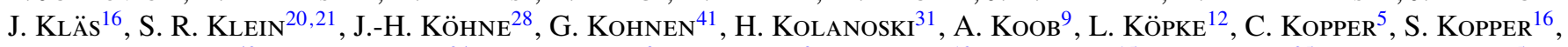

D. J. Koskinen ${ }^{42}$, M. Kowalski ${ }^{24}$, A. Kriesten ${ }^{9}$, K. Krings 9 , G. Kroll ${ }^{12}$, M. Kroll ${ }^{15}$, J. Kunnen ${ }^{25}$, N. Kurahashi ${ }^{5}$,

T. Kuwabara ${ }^{32}$, M. Labare ${ }^{30}$, D. T. Larsen ${ }^{5}$, M. J. Larson ${ }^{42}$, M. Lesiak-BzdaK ${ }^{40}$, M. LeuermanN ${ }^{9}$, J. Leute ${ }^{18}$,

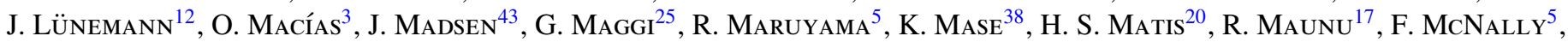
K. Meagher ${ }^{17}$, M. Medici ${ }^{42}$, A. Meli ${ }^{30}$, T. Meures ${ }^{36}$, S. MiareCKi $^{20,21}$, E. Middell ${ }^{2}$, E. Middlemas ${ }^{5}$, N. Milke ${ }^{28}$, J. Miller $^{25}$,

L. Mohrmann ${ }^{2}$, T. Montaruli ${ }^{4}$, R. Morse ${ }^{5}$, R. Nahnhauer ${ }^{2}$, U. Naumann ${ }^{16}$, H. Niederhausen ${ }^{40}$, S. C. Nowicki ${ }^{35}$,

D. R. Nygren ${ }^{20}$, A. Obertacke ${ }^{16}$, S. Odrowski ${ }^{35}$, A. Olivas $^{17}$, A. Omairat ${ }^{16}$, A. O’Murchadha ${ }^{36}$, T. Palczewski ${ }^{44}$, L. Paul $^{9}$,

Ö. Penek ${ }^{9}$, J. A. Pepper ${ }^{44}$, C. Pérez de los Heros ${ }^{22}$, C. Pfendner ${ }^{13}$, D. Pieloth ${ }^{28}$, E. Pinat ${ }^{36}$, J. Posselt ${ }^{16}$, P. B. Price ${ }^{21}$,

G. T. Przybylski ${ }^{20}$, J. PÜtz ${ }^{9}$, M. Quinnan ${ }^{8}$, L. RÄdel ${ }^{9}$, M. Rameez $^{4}$, K. Rawlins ${ }^{45}$, P. RedL ${ }^{17}$, I. Rees ${ }^{5}$, R. ReimanN ${ }^{9}$,

E. Resconi ${ }^{18}$, W. Rhode ${ }^{28}$, M. Richman ${ }^{17}$, B. Riedel ${ }^{5}$, S. Robertson ${ }^{1}$, J. P. Rodrigues ${ }^{5}$, M. Rongen ${ }^{9}$, C. RotT ${ }^{23}$, T. Ruhe ${ }^{28}$,

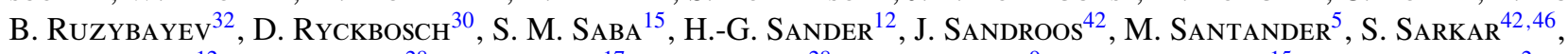

K. Schatto ${ }^{12}$, F. Scheriad ${ }^{28}$, T. Schmidt ${ }^{17}$, M. Schmitz ${ }^{28}$, S. Schoenen ${ }^{9}$, S. Schöneberg ${ }^{15}$, A. Schönwald ${ }^{2}$,

A. Schukraft ${ }^{9}$, L. Schulte ${ }^{24}$, O. Schulz ${ }^{18}$, D. Seckel ${ }^{32}$, Y. Sestayo ${ }^{18}$, S. Seunarine $^{43}$, R. Shanidze ${ }^{2}$, C. Sheremata $^{35}$, M. W. E. Smith ${ }^{8}$, D. Soldin ${ }^{16}$, G. M. SpiczaK ${ }^{43}$, C. Spiering ${ }^{2}$, M. Stamatikos ${ }^{13,47}$, T. Stanev ${ }^{32}$, N. A. Stanisha ${ }^{8}$, A. StasiK ${ }^{24}$, T. Stezelberger ${ }^{20}$, R. G. Stokstad ${ }^{20}$, A. StößL ${ }^{2}$, E. A. Strahler ${ }^{25}$, R. Ström ${ }^{22}$, N. L. Strotjohann ${ }^{24}$, G. W. Sullivan ${ }^{17}$,

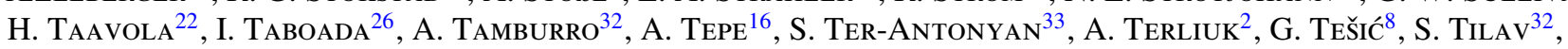

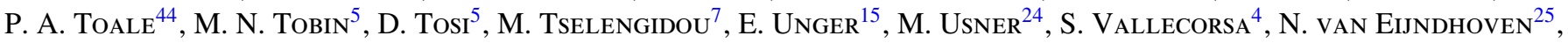

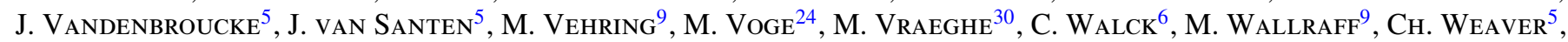
M. Wellons ${ }^{5}$, C. Wendt ${ }^{5}$, S. Westerhoff 5 , B. J. Whelan ${ }^{1}$, N. Whitehorn ${ }^{5}$, C. Wichari ${ }^{9}$, K. Wiebe ${ }^{12}$, C. H. Wiebusch ${ }^{9}$, D. R. Williams ${ }^{44}$, H. Wissing ${ }^{17}$, M. Wolf ${ }^{6}$, T. R. Wood ${ }^{35}$, K. WoschnaGG ${ }^{21}$, D. L. Xu ${ }^{44}$, X. W. XU ${ }^{33}$, J. P. YaneZ ${ }^{2}$, G. YodH $^{11}$, S. Yoshida ${ }^{38}$, P. ZarZhitsky ${ }^{44}$, J. ZiemanN ${ }^{28}$, S. Zierke ${ }^{9}$, AND M. ZOLL ${ }^{6}$

(ICeCube Collaboration)

${ }^{1}$ School of Chemistry and Physics, University of Adelaide, Adelaide SA, 5005 Australia ${ }^{2}$ DESY, D-15735 Zeuthen, Germany

${ }^{3}$ Department of Physics and Astronomy, University of Canterbury, Private Bag 4800, Christchurch, New Zealand

${ }^{4}$ Département de physique Nucléaire et Corpusculaire, Université de Genève, CH-1211 Genève, Switzerland

${ }^{5}$ Department of Physics and Wisconsin IceCube Particle Astrophysics Center, University of Wisconsin, Madison, WI 53706, USA

${ }^{6}$ Oskar Klein Centre and Department of Physics, Stockholm University, SE-10691 Stockholm, Sweden

${ }^{7}$ Erlangen Centre for Astroparticle Physics, Friedrich-Alexander-Universität Erlangen-Nürnberg, D-91058 Erlangen, Germany

${ }^{8}$ Department of Physics, Pennsylvania State University, University Park, PA 16802, USA

${ }^{9}$ III. Physikalisches Institut, RWTH Aachen University, D-52056 Aachen, Germany

${ }^{10}$ Physics Department, South Dakota School of Mines and Technology, Rapid City, SD 57701, USA

${ }^{11}$ Department of Physics and Astronomy, University of California, Irvine, CA 92697, USA

${ }^{12}$ Institute of Physics, University of Mainz, Staudinger Weg 7, D-55099 Mainz, Germany

${ }^{13}$ Department of Physics and Center for Cosmology and Astro-Particle Physics, Ohio State University, Columbus, OH 43210, USA ${ }^{14}$ Department of Astronomy, Ohio State University, Columbus, OH 43210, USA

${ }^{15}$ Fakultät für Physik and Astronomie, Ruhr-Universität Bochum, D-44780 Bochum, Germany

${ }_{17}^{16}$ Department of Physics, University of Wuppertal, D-42119 Wuppertal, Germany

${ }^{17}$ Department of Physics, University of Maryland, College Park, MD 20742, USA

${ }^{18}$ Technische Universität München, D-85748 Garching, Germany

${ }^{19}$ Department of Physics and Astronomy, University of Kansas, Lawrence, KS 66045, USA 


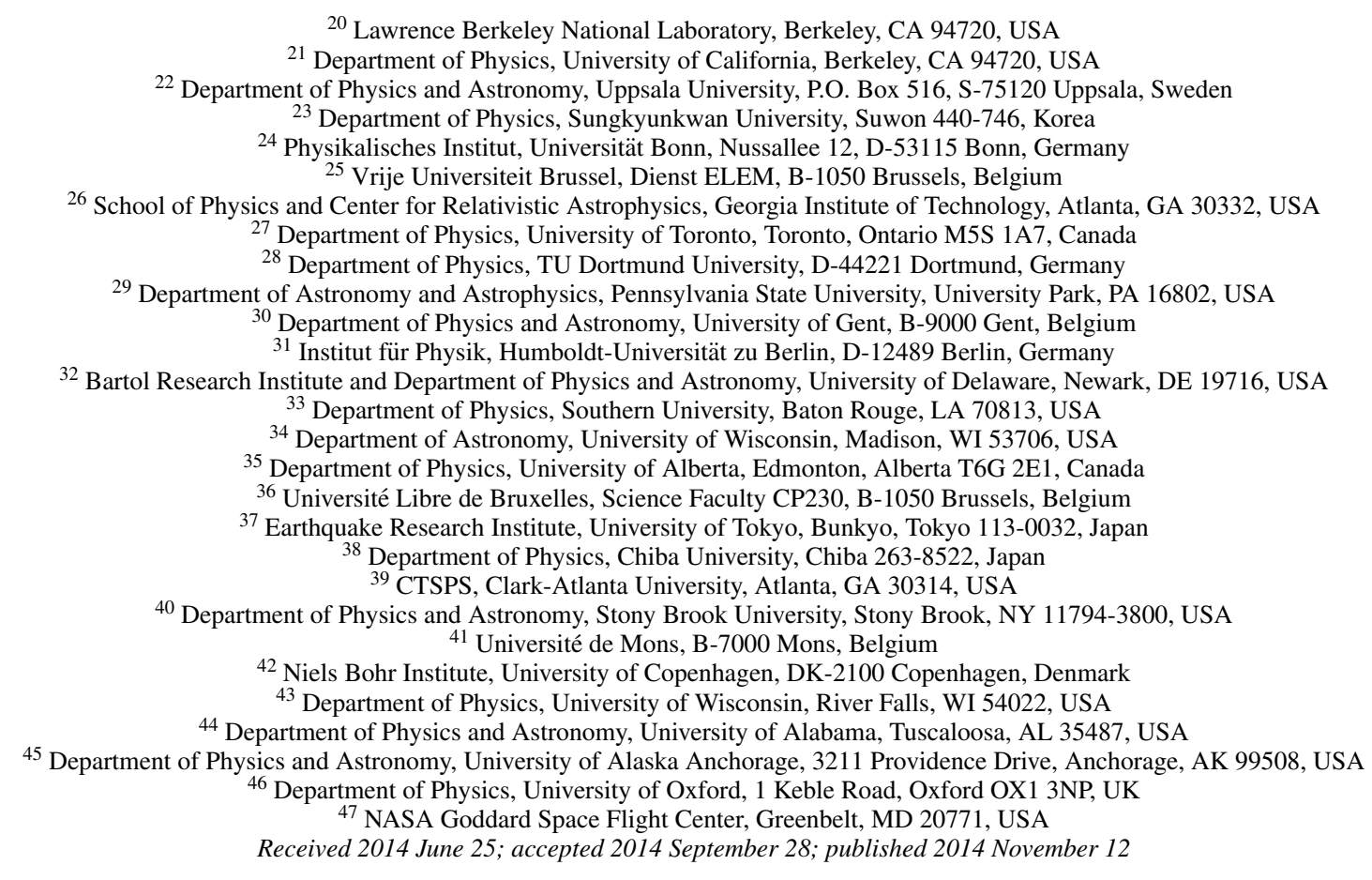

\begin{abstract}
We present results on searches for point-like sources of neutrinos using four years of IceCube data, including the first year of data from the completed 86 string detector. The total livetime of the combined data set is 1373 days. For an $E^{-2}$ spectrum, the observed 90\% C.L. flux upper limits are $\sim 10^{-12} \mathrm{TeV}^{-1} \mathrm{~cm}^{-2} \mathrm{~s}^{-1}$ for energies between $1 \mathrm{TeV}$ and $1 \mathrm{PeV}$ in the northern sky and $\sim 10^{-11} \mathrm{TeV}^{-1} \mathrm{~cm}^{-2} \mathrm{~s}^{-1}$ for energies between $100 \mathrm{TeV}$ and $100 \mathrm{PeV}$ in the southern sky. This represents a $40 \%$ improvement compared to previous publications, resulting from both the additional year of data and the introduction of improved reconstructions. In addition, we present the first results from an all-sky search for extended sources of neutrinos. We update the results of searches for neutrino emission from stacked catalogs of sources and test five new catalogs; two of Galactic supernova remnants and three of active galactic nuclei. In all cases, the data are compatible with the background-only hypothesis, and upper limits on the flux of muon neutrinos are reported for the sources considered.
\end{abstract}

Key words: astroparticle physics - galaxies: active - galaxies: clusters: general - galaxies: starburst ISM: supernova remnants - neutrinos

Online-only material: color figures

\section{INTRODUCTION}

Neutrinos have unique properties that can be used to probe diverse astrophysical processes. Produced in interactions of protons and nuclei with ambient radiation and matter, their low cross section allows them to travel astronomical distances without experiencing significant absorption. Unlike charged cosmic rays (CRs) which change direction as they pass through galactic and intergalactic magnetic fields, neutrinos preserve their directional information as they travel straight from the source to Earth. Astrophysical neutrinos are also tracers of hadronic interactions, and the identification of these neutrino sources may help to clarify CR acceleration processes (Anchordoqui \& Montaruli 2010; Anchordoqui et al. 2014; Becker 2008; Halzen \& Hooper 2002; Learned \& Mannheim 2000). Candidate sources for CR acceleration (and therefore neutrino emission) include supernova remnant (SNR) shocks (Alvarez-Muñiz \& Halzen 2002; Cavasinni et al. 2006; Gonzalez-Garcia et al. 2014; De Marco et al. 2006; Vissani et al. 2011), active galactic nuclei (AGNs) jets (Essey et al. 2010; Kalashev et al. 2013; Murase et al. 2014; Sironi \& Spitkovsky 2011; Stecker et al. 1991; Waxman
\& Bahcall 1999), starburst galaxies (Lacki et al. 2011; Loeb \& Waxman 2006; Murase et al. 2013; Romero \& Torres 2003), and gamma-ray bursts (Guetta et al. 2004; Mészáros 2006; Waxman $\&$ Bahcall 1997).

IceCube recently found evidence for a diffuse flux of highenergy astrophysical neutrinos (Aartsen et al. 2013e, 2014b), observing a $5.7 \sigma$ excess of events between $\sim 50 \mathrm{TeV}$ and $2 \mathrm{PeV}$ deposited within the detector. The 37 observed events are consistent with an $E^{-2.3}$ neutrino flux at the level of $1.5 \times 10^{-11} \mathrm{TeV}^{-1} \mathrm{~cm}^{-2} \mathrm{~s}^{-1} \mathrm{sr}^{-1}$ (normalized at $100 \mathrm{TeV}$ ), with a neutrino flavor ratio of 1:1:1. While these events have established unequivocally that astrophysical neutrinos exist, their sources have not yet been identified. One challenge is that only $\sim 20 \%$ of the events in that sample are associated with a high-energy muon which leaves a visible track in the detector. The remaining events without a track have a poor angular resolution of $\sim 15^{\circ}$.

This paper presents the latest results of searches for point sources of astrophysical neutrinos with a sample of track-like events associated with $v_{\mu}$ (and some $v_{\tau}$ ) charged current interactions observed by the IceCube Neutrino Observatory. 
These events have an excellent angular resolution of $\leqslant 1^{\circ}$ and hence allow us to point back toward the source. As the main signature we focus on is the resultant muon, the interaction vertex is not required to lie inside the detector as in Aartsen et al. (2013e, 2014b) and the effective volume is hence effectively enhanced. The results of an all-sky search, a search among a catalog of candidate neutrino emitters and stacked source catalog searches with a similar sample of events from the data collected between 2008 and 2011, are published in Aartsen et al. (2013c). Here we update these analyses by adding the first year of data from the complete 86 string detector configuration, collected between 2011 May and 2012 May. Five new stacking analyses based on newly available catalogs are also presented here.

In this paper, we describe the results of the first all-sky survey by IceCube looking for extended regions of neutrino emission. H.E.S.S. has surveyed the Galactic plane looking for gammaray emissions above $200 \mathrm{GeV}$, revealing previously unknown extended regions emitting to TeV energies (Carrigan et al. 2013). The Fermi/Large Area Telescope (LAT) survey above $100 \mathrm{GeV}$ also shows the same bright extended sources. These extended regions may be unidentified SNRs associated with molecular clouds, which are also expected to be spatially extended sources of neutrinos (Gonzalez-Garcia et al. 2014; Mandelartz \& Tjus 2013). Outside the Galaxy, large clusters of galaxies such as Virgo are promising neutrino emitters expected to have spatial extensions (De Marco et al. 2006; Murase et al. 2008; Murase \& Beacom 2012; Wolfe et al. 2008). It is therefore important not to limit the search for sources of neutrinos uniquely to point-like sources, but also to extended regions as shown in Tchernin et al. (2013).

Section 2 describes the IceCube detector and the event selection for data from the first year of the completed detector. Event selections for data from the previous years of operation of the detector have been extensively described in Aartsen et al. (2013c) and Abbasi et al. (2011). The methodology used to optimize the searches for various source signal hypotheses is described in Section 3. Section 4 presents the results of the analyses, which are discussed within the context of recent models of astrophysical neutrino emission. Conclusions are drawn in Section 5.

\section{DETECTOR AND EVENT SELECTION}

The IceCube Observatory is a cubic-kilometer-sized Cherenkov detector embedded in the ice at the geographic south pole (Achterberg et al. 2006). Optimized to detect neutrinos above $\mathrm{TeV}$ energies, it consists of 5160 photomultiplier tubes (PMTs) instrumented along 86 cables (called strings) at depths of 1450-2450 m beneath the surface of the ice sheet. Each PMT is housed in a digital optical module (DOM), consisting of a pressure-resistant sphere with on board digitization and calibration LEDs (light-emitting diodes; Abbasi et al. 2010). The DOMs detect Cherenkov photons emitted by charged leptons that traverse the detector (Abbasi et al. 2009). This analysis uses data taken between 2008 April and 2012 May. During this period, IceCube ran in four different configurations. Three years of data are from the partial detector composed of 40, 59, and 79 strings, respectively, and are fully described in Aartsen et al. (2013c). The following year of data was taken with the completed 86 string array. The used selection procedure and event reconstructions are similar to those applied to the previous data.

\subsection{Data Reduction and Reconstruction for the IC86-1 Data Sample}

Data acquisition is triggered by requiring four pairs of neighboring or next-to-neighboring DOMs to observe photoelectrons within a $5 \mu$ s time window. $2.5 \mathrm{kHz}$ of data satisfy this criterion. A combination of real-time filtering at the south pole and subsequent offline CPU-intensive processing reduces the data rate to $2 \mathrm{~Hz}$ by rejecting misreconstructed events. At this stage, the data are dominated by atmospheric muons from CRs; both well-reconstructed down-going muons in the southern sky and down-going muons misreconstructed as up-going muons in the northern sky. The data is further reduced via quality cuts using simple reconstructions and event quality parameters followed by advanced likelihood-based muon reconstructions. The simple reconstruction removes scattered photon hits before estimating the muon position and direction via a linear fit with reduced weights for outliers (Aartsen et al. 2013b). This fit serves as a seed for more advanced likelihood reconstructions, including the multi-photoelectron (MPE) likelihood. This algorithm includes a probabilistic distribution function (PDF), which describes the scattering of photons in the ice, and is fully described in Ahrens et al. (2004).

In the processing of data from the first year of the full detector, two new muon reconstructions were used to determine event directions and reject background. The first reconstructs the muon direction by applying the MPE likelihood four times. Each iteration uses a bootstrapped pulse series, extracted randomly from the measured pulses. This is done using a multinomial distribution weighted by charge, so that high-charge pulses are more likely to be selected than low ones. The results of these four reconstructions are averaged together to seed one reconstruction using the complete pulses. Of these five fit results, the one with the best likelihood value is selected and saved. Compared to the single-iteration MPE fit, this process reduces the rate of down-going atmospheric muons misreconstructed as up-going muons by $30 \%$, while improving the neutrino median angular resolution from 0.7 to 0.6 at $30 \mathrm{TeV}$.

This iterative fit also serves as a seed for the second reconstruction algorithm, which provides a more accurate result by modeling the optical properties of the Antarctic ice sheet. While previous reconstructions use analytic approximations to describe the timing distribution of Cherenkov photons arriving at a given PMT (Ahrens et al. 2004), here we use a parameterization of a Monte Carlo simulation. Photon transport is simulated using a depth-dependent model of scattering and absorption in the ice (Aartsen et al. 2013a). The arrival time of a photon is a function of the orientation and depth of the muon source and the displacement vector between the muon and the receiving PMT. Photons are simulated for different muon-receiver configurations, and a multi-dimensional spline surface is fit to the resulting arrival time distributions (Whitehorn et al. 2013). These splines are used as PDFs in the MPE likelihood. Compared to previous IceCube point source analyses (Aartsen et al. 2013c), this reconstruction algorithm leads to a $26 \%$ improvement in neutrino median angular resolution at $30 \mathrm{TeV}$ (see Figure 1). As carried out in previous years, the uncertainty in the angular reconstruction for each event is estimated by fitting a paraboloid to the likelihood space around the reconstructed direction, following the method described in Abbasi et al. (2011) and Neunhöffer (2006).

After reconstructing the direction of each event, a separate algorithm fits for the muon energy loss along its track. In the fourth year of data, the energy reconstruction uses an analytic 


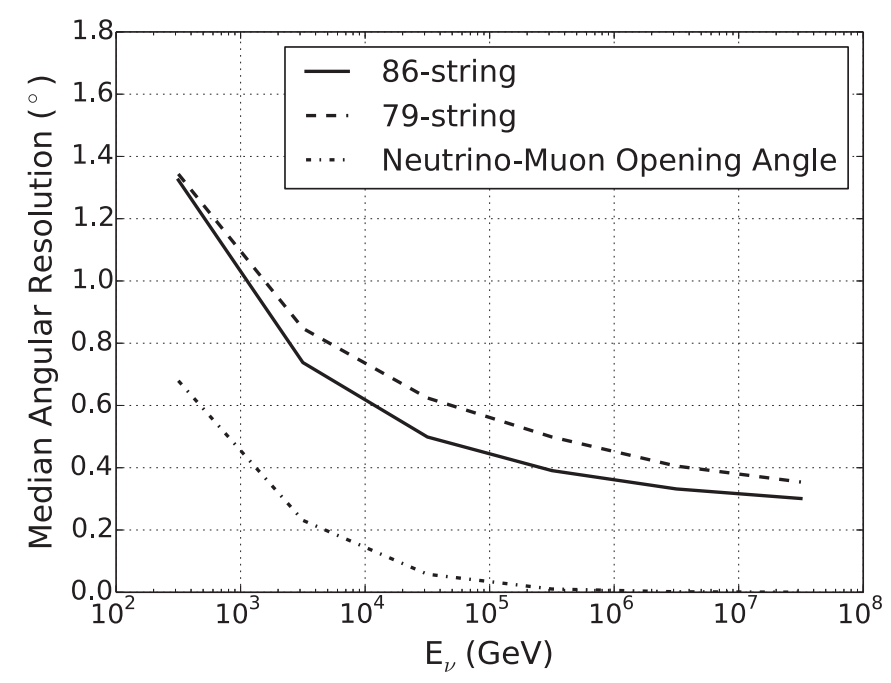

Figure 1. Median angular resolution (angle between reconstructed muon track and neutrino direction) as a function of neutrino energy for simulated northern hemisphere event samples from the 86 string (solid) and 79 string (dashed) detector configurations. The improvement is due to the new reconstruction algorithm. At $30 \mathrm{TeV}$, the 40 and 59 string event selections (not shown) give angular resolutions of $\sim 0.8$ and $\sim 0.75$, respectively (Aartsen et al. 2013c). The dash-dotted line shows the median kinematic opening angle between the neutrino and muon.

approximation to model the muon light yield at the receiving DOMs as a function of the orientation and depth of the muon (Aartsen et al. 2014a).

\subsection{Selection of the Final Sample}

From the $2 \mathrm{~Hz}$ of remaining data (still dominated by the atmospheric muon background), $4.8 \mathrm{mHz}$ of events are selected for the final analysis sample. In the northern sky, the misreconstructed muon background can be mostly eradicated to isolate a nearly pure sample of up-going atmospheric neutrinos. This is done using a classification algorithm called boosted decision trees (BDTs). Similar to previous IceCube point source analyses (Aartsen et al. 2013c), we trained four BDTs in two zenith bands to separate astrophysical neutrino signal from the atmospheric muon background. Cuts on the BDT output scores are optimized to achieve the best discovery potential for both $E^{-2}$ and $E^{-2.7}$ signal spectra. This event selection covers the entire northern hemisphere and extends $5^{\circ}$ above the horizon, where the Earth and glacial ice still provide a shield from the CR background.

At an angle of more than $5^{\circ}$ above the horizon, a pure neutrino sample cannot be isolated from the high-energy atmospheric muon bundles, which are multiple muons from the same air shower that mimic neutrinos. The background can be reduced by introducing quality cuts and using parameters that select neutrinos and reject muon bundles. One BDT is trained for the entire region using data to describe the background and an $E^{-2}$ neutrino simulation for signal. Of the 11 variables used in training the BDT, 3 exploit differences between single muons and bundles. These parameters rely on event topology and energy loss information. Large muon bundles consist of many low-energy muons that typically lose energy at a constant rate as they traverse the detector. Photons from these muon bundles are detected within a wider time range. High-energy, neutrinoinduced muons instead have relatively stochastic energy loss profiles and narrower photon timing distributions. Likelihood ratios are constructed to judge whether a given data event has timing and energy loss properties more consistent with the
Table 1

Summary for Four Different IceCube Configurations for Point Source Analyses: the Expected Atmospheric Neutrino Rate from MC Simulation Weighted for the Model in Honda et al. (2007) and Numbers of Up- and Down-going Events at Final Selection Level

\begin{tabular}{lcccc}
\hline \hline No. of Strings & $\begin{array}{c}\text { Livetime } \\
(\text { days })\end{array}$ & $\begin{array}{c}\text { Atm. vs } \\
\left(\text { day }^{-1}\right)\end{array}$ & No. of Up-going & No. of Down-going \\
\hline 40 & 376 & 40 & 14121 & 22779 \\
59 & 348 & 120 & 43339 & 64230 \\
79 & 316 & 180 & 50857 & 59009 \\
86 & 333 & 210 & 69227 & 69095 \\
\hline
\end{tabular}

Note. The up-going data are dominated by atmospheric neutrinos, while data in the down-going region are dominated by atmospheric muons.

simulated signal or the estimated background and are included in the BDT. To obtain the final sample, a cut on the BDT score is varied with zenith to account for the zenith-dependent properties of the background.

The final data sample for the first year of operation of the 86 string detector has 138,322 events, of which approximately half are in the northern hemisphere. The livetime and rates for all four years of detector data are summarized in Table 1. The neutrino effective area for this selection and the central $90 \%$ energy region for three signal spectra are shown in Figure 2. The effective area reaches its maximum near the horizon. Far below the horizon, high-energy neutrinos suffer from absorption in the Earth. Above the horizon, the cuts necessary to remove the background remove a significant portion of the lowerenergy signal. As a result, the analysis is sensitive to the widest neutrino energy range near the horizon, while in the southern hemisphere the sensitivity rapidly deteriorates at lower energies. The discovery potential as a function of energy and declination (decl.) is shown in Figure 3. The discovery potential is defined as the flux required to observe a $5 \sigma$ upward fluctuation before trials, while the analysis sensitivity is defined as the median upper limit observed in the absence of a signal. Compared to the three year point source analysis (Aartsen et al. 2013c), the addition of the first year of data from the completed detector including improved reconstruction and background rejection techniques leads to a $40 \%-50 \%$ improvement in both the sensitivity and discovery potential, with larger gains at energies below $1 \mathrm{PeV}$ in the southern hemisphere.

\section{THE LIKELIHOOD SEARCH METHOD}

Point-like sources of neutrinos in the sky can be identified by searching for clusters of events significantly incompatible with the atmospheric muon and neutrino background. The significance is estimated by using an unbinned maximum likelihood ratio test as described in Braun et al. (2010). The method is expanded to allow for the combination of data from different detector geometries as described in Aartsen et al. (2013c). In addition to spatial clustering, this method also uses the energies of the events to identify signal events that are expected to have a harder spectrum than that of atmospheric neutrinos and muons. The energy response expected from a neutrino signal from a point source in the sky is modeled using simulation. Since the final event selections are still backgrounddominated, the background estimate is done using real data.

In time-integrated searches for a point-like source, the signal PDF $\mathcal{S}_{i}^{j}$ for event $i$ observed in detector geometry $j$ is given by

$$
\mathcal{S}_{i}^{j}=S_{i}^{j}\left(\left|\boldsymbol{x}_{i}-\boldsymbol{x}_{s}\right|, \sigma_{i}\right) \mathcal{E}_{i}^{j}\left(E_{i}, \delta_{i}, \gamma\right)
$$



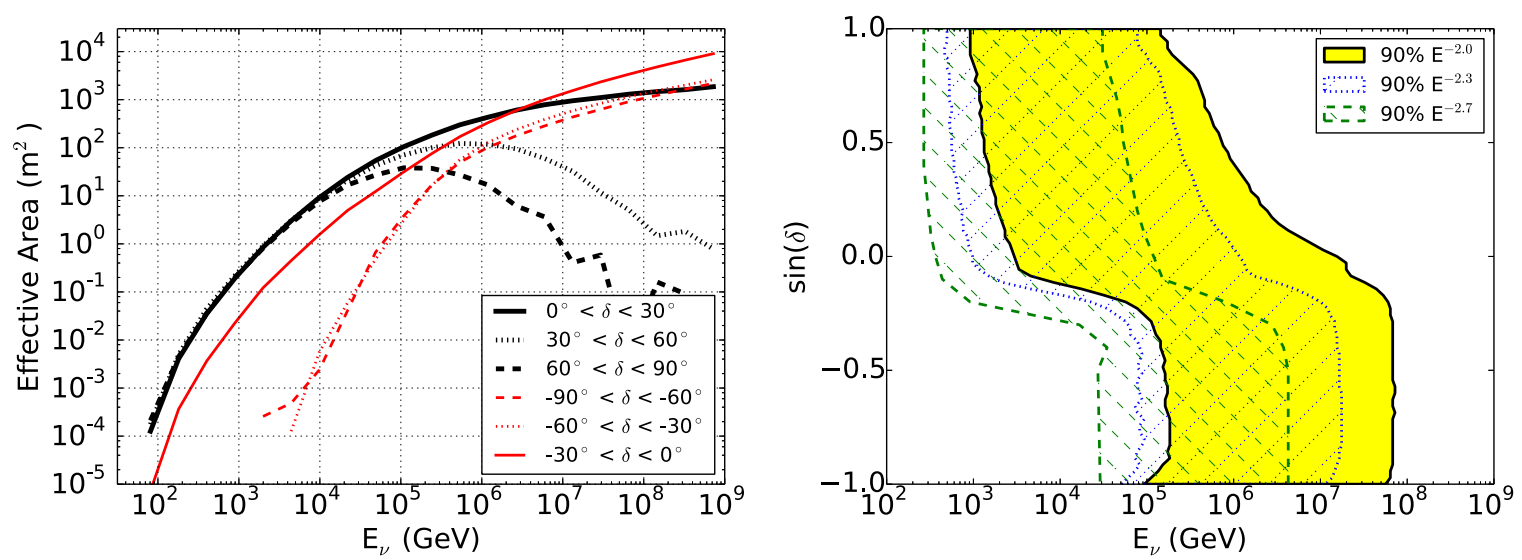

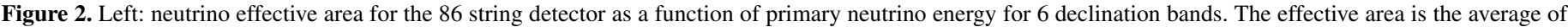

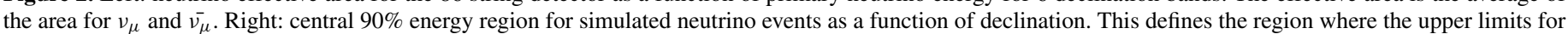
$E^{-2}, E^{-2.3}$, and $E^{-2.7}$ source spectra are valid.

(A color version of this figure is available in the online journal.)

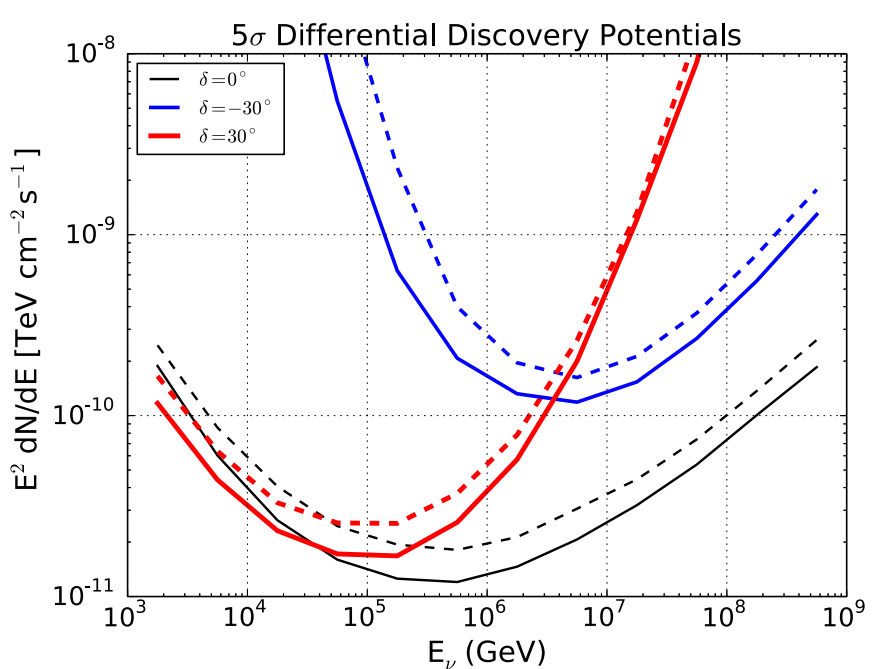

Figure 3. Discovery flux as a function of the neutrino energy at a $5 \sigma$ confidence level for three different declinations (solid lines). Point sources with an $E^{-2}$ spectrum are simulated over a half decade in energy, and the flux in each bin required for discovery forms the curve above. Results from the previous analysis with three years of the data are shown with dashed lines.

(A color version of this figure is available in the online journal.)

Here, the spatial contribution to the PDF is given by $S_{i}^{j}$, which depends on the angular uncertainty of the event $\sigma_{i}$, and the angular difference between the reconstructed direction of the event and the direction of the source. This probability is modeled as a two-dimensional Gaussian:

$$
S_{i}^{j}=\frac{1}{2 \pi \sigma_{i}^{2}} e^{-\frac{\left|x_{i}-x_{s}\right|^{2}}{2 \sigma_{i}^{2}}} .
$$

The contribution from energy $\mathcal{E}_{i}^{j}\left(E_{i}, \delta_{i}, \gamma\right)$ is described in Braun et al. (2010).

When searching for spatially extended sources, the value of $\sigma_{i}$ is replaced with $\sigma_{i}^{\text {eff }}=\sqrt{\sigma_{i}^{2}+\sigma_{\text {stc }}^{2}}$, where $\sigma_{\text {src }}$ is the width of the source. Figure 4 shows the flux needed for a $5 \sigma$ discovery for a source located at a given declination as a function of the source extension. The results for two different signal hypotheses are shown; in one, the source is always assumed to have no extension, while in the other the correct source extension is included in the likelihood description. Naturally, for

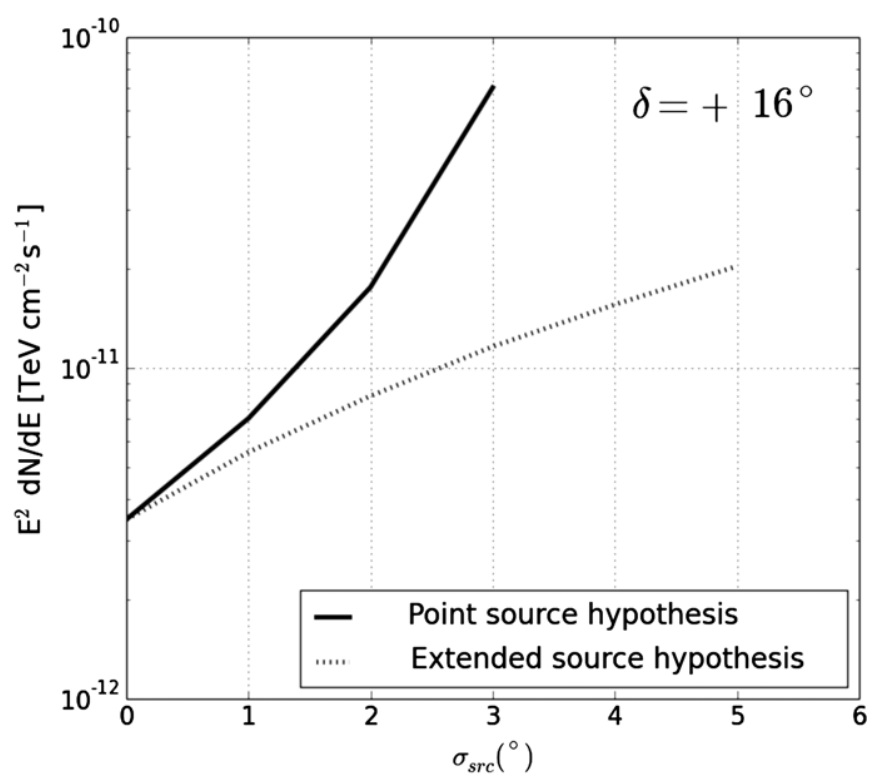

Figure 4. Flux needed for a $5 \sigma$ discovery from a hypothetical source at $\delta=16^{\circ}$ as a function of the true source extension for the point source signal hypothesis (solid line) and the extended signal hypothesis with the correct extension (dotted line). For a source extended by $3^{\circ}$, a search using the simple point source signal $\mathrm{PDF}$ of Equation (2) requires a flux $\sim$ six times larger than a search incorporating the true extension of the source within the signal PDF to produce a $5 \sigma$ discovery. A point source search can be viewed as a special case of an extended source search with $\sigma_{\mathrm{src}}=0$. When the true extension of a source varies from one of the five extensions assumed in the search hypotheses by up to 0.5 , the flux required for discovery is expected to vary by $\sim 10 \%$.

sources that are truly extended, the extended hypothesis is more powerful than the point source assumption. As the real extension of the source increases, the analysis method which assumes that the source is point-like performs worse than the one that takes the extension of the source in to account.

To further enhance discovery potentials and sensitivity, stacked searches can be carried out for specific catalogs of similar candidate neutrino sources.

The following is a description of all the searches performed with the four years of IceCube data (similar to those performed in Aartsen et al. 2013c). 

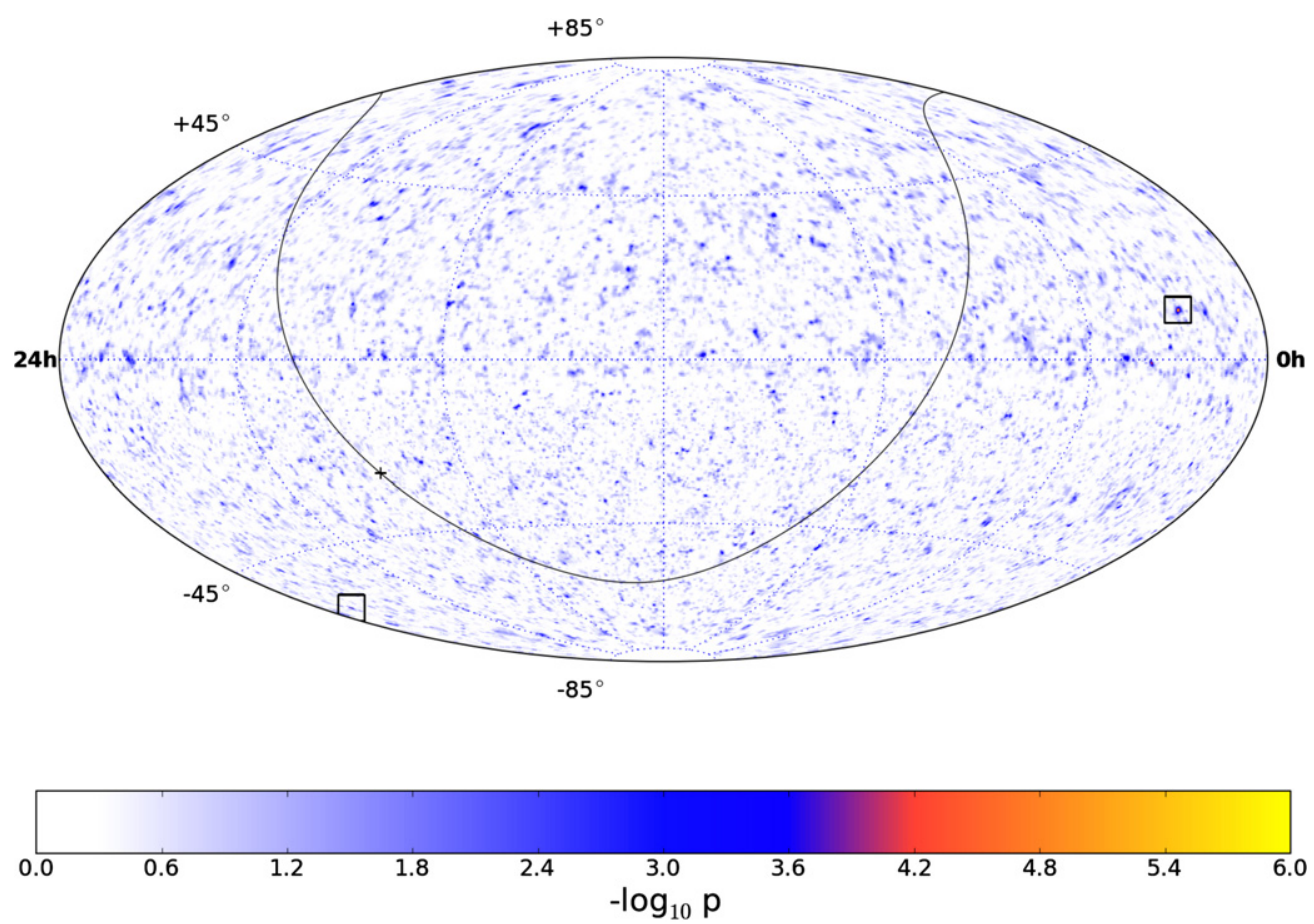

Figure 5. Pre-trial significance skymap in equatorial coordinates (J2000) of the all-sky point source scan for the combined four year data sample. The black line indicates the Galactic plane, and the black plus sign indicates the Galactic center. The most significant fluctuation in each hemisphere is indicated with a square marker. (A color version of this figure is available in the online journal.)

Table 2

Summary of the Results from the Extended All-sky Survey

\begin{tabular}{lrrrccc}
\hline \hline Extension $\left({ }^{\circ}\right)$ & R.A. $\left({ }^{\circ}\right)$ & Decl. $\left({ }^{\circ}\right)$ & $\hat{n}_{S}$ & $\hat{\gamma}$ & $p$-value (Pre-trial) & $p$-value (Post-trial) \\
\hline $1^{\circ}$ & 286.25 & -43.25 & 49.6 & 2.65 & $6.75 \times 10^{-5}$ & 0.58 \\
$2^{\circ}$ & 248.75 & 62.75 & 58.2 & 2.38 & $5.52 \times 10^{-4}$ & 0.87 \\
$3^{\circ}$ & 30.75 & -30.25 & 93.6 & 3.10 & $1.22 \times 10^{-3}$ & 0.81 \\
$4^{\circ}$ & 30.75 & -30.25 & 99 & 3.10 & $3.29 \times 10^{-3}$ & 0.81 \\
$5^{\circ}$ & 251.75 & 61.25 & 102 & 2.54 & $1.06 \times 10^{-2}$ & 0.91 \\
\hline
\end{tabular}

Note. The coordinates of the most significant spots located for each source extension hypothesis are given together with the respective $p$-values.

\subsection{All-sky Searches}

These searches are carried out to look for evidence of a source anywhere in the sky and are not motivated by any prior information regarding the position of the sources. The likelihood is evaluated in each direction in the sky. In these searches, the number of effective trials is very high and is related to the angular resolution of the telescope and the source extension hypotheses. In order to correct for the trial factor, the same experiment is repeated on an ensemble of scrambled data and the probability of observing a more significant spot than the one observed is obtained.

All-sky point source scan. The all-sky scan for point sources of neutrinos that has previously been carried out on data from the incomplete detector configurations is updated to include the first year of data from the complete 86 string detector. In this search, the likelihood is evaluated in steps of $0.1 \times 0.1$ within the declination range $-85^{\circ}$ to $+85^{\circ}$. We do not search for point sources at declinations $|\delta|>85^{\circ}$ because there is not sufficient phase space in right ascension (R.A.) to estimate significances with the scrambling technique.
All-sky extended source scans. The search for extended sources is performed in a similar fashion to the all-sky point source searches. In this case, the sky is divided into a grid of $0.5 \times 0.5$ in a similar declination range. For this search, a source extension needs to be assumed for the signal. We carry out five different all-sky scans assuming extensions in step of $1^{\circ}$, from $1^{\circ}$ to $5^{\circ}$. An additional trial factor needs to be considered from the additional number of sky scans; however, this factor can be conservatively assumed to be five.

\subsection{Searches Among List of 44 Candidate Sources}

In order to reduce the large number of effective trials associated with scanning the entire sky, we also performed a search for the most significant of 44 a priori selected source candidates. The sources in this list have been selected according to observations in gamma-rays or astrophysical models predicting neutrino emission.

\subsection{Stacking Searches}

Several sources of the same type may emit fluxes that are individually below the discovery potential but detectable 

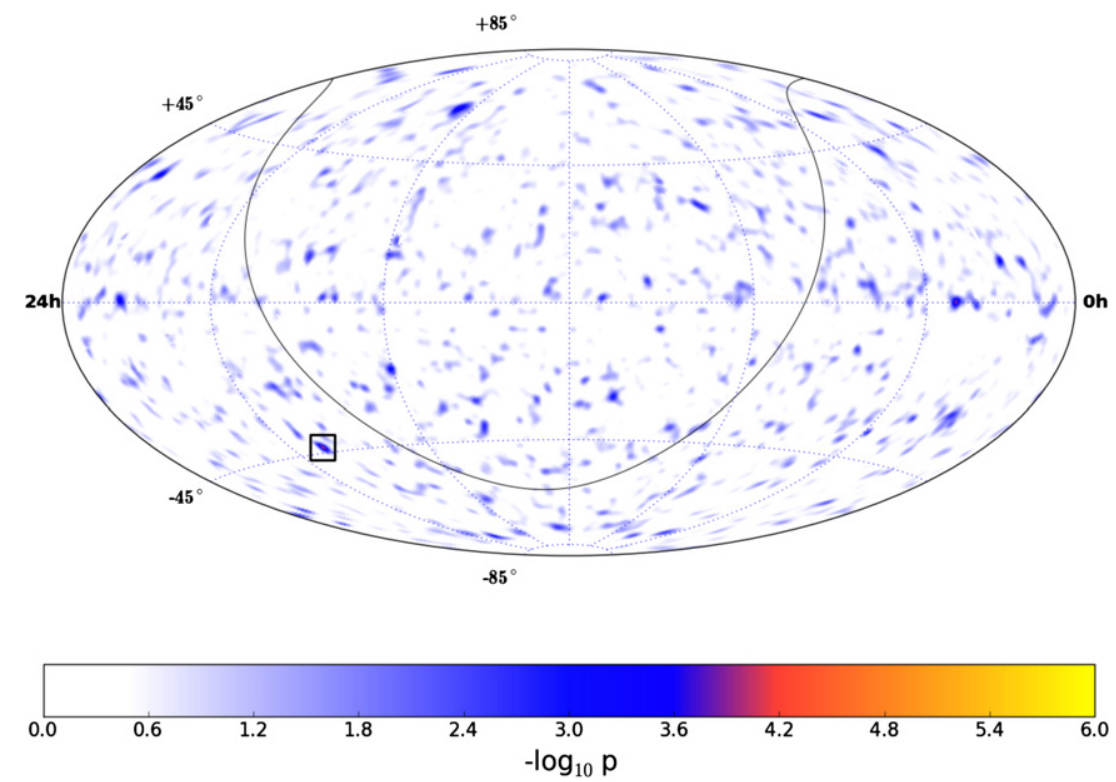

Figure 6. Pre-trial significance skymap from the all-sky scan for sources of $1^{\circ}$ extension in equatorial coordinates. The black line indicates the Galactic plane. The most significant fluctuation is indicated with a square marker.

(A color version of this figure is available in the online journal.)
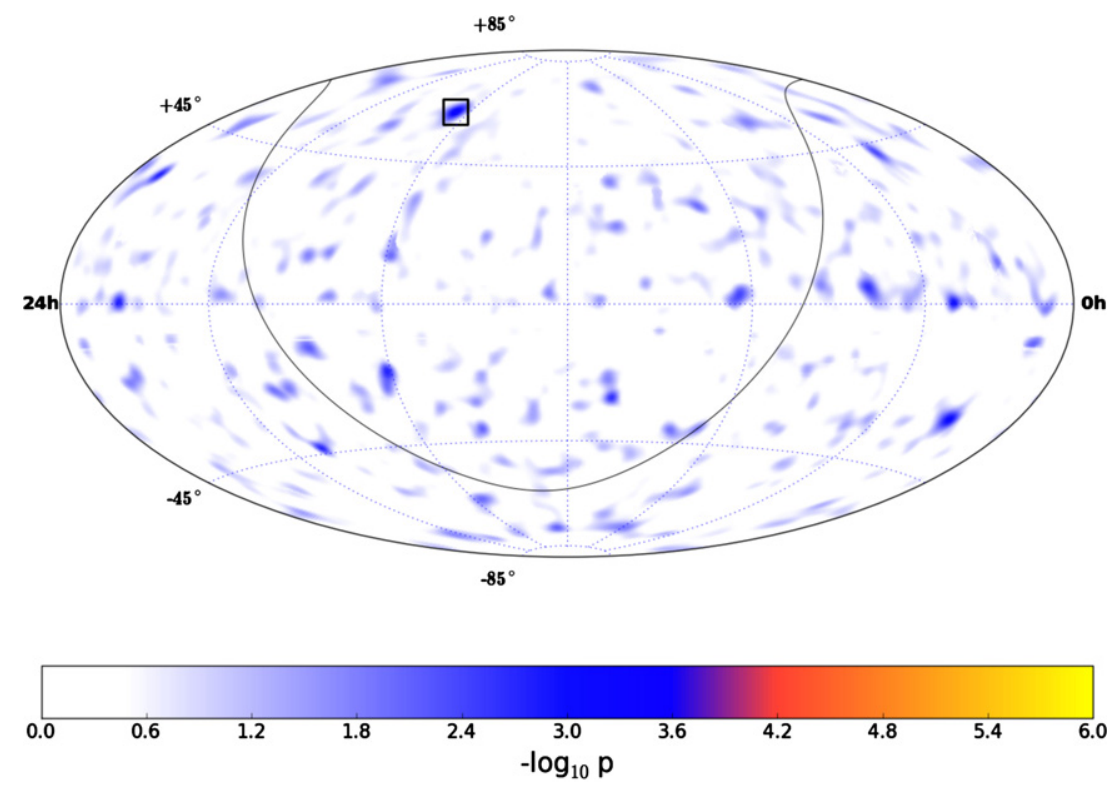

Figure 7. Same as Figure 6, but for sources of $2^{\circ}$ extension.

(A color version of this figure is available in the online journal.)

as a class when summed up using the stacking technique. Here we report on the different catalogs of sources that have similar spectral behavior based on gamma-ray observations or astrophysical models predicting neutrino emission. For these searches, the signal PDF $\mathcal{S}_{i}^{j}$ of Equation (1) is modified to accommodate multiple sources (see Abbasi et al. 2011). A prior knowledge of the expected luminosities of these sources can be utilized to weight the contribution of each source in the total signal PDF to make the search optimal for that signal hypothesis. Alternatively, an equal-weighting can be applied if there is no preferred model. In the following section, we summarize all the stacking searches performed with four years of data. Most of these searches are updates from the previous results using three years of data (Aartsen et al. 2013c).
Updated searches. These searches have been previously carried out on three years of data (Aartsen et al. 2013c) and are now updated to include data from the first year of operation of the completed 86 string detector.

Six Milagro TeV gamma-ray sources. The authors of the model that motivated the original analysis have hence updated the models to reflect the newer gamma-ray observations (Gonzalez-Garcia et al. 2014). For this reason, in this search an equal weight is used for each source in the likelihood with the intention of keeping our sensitivity optimal for all possible signal hypothesis.

One hundred and twenty-seven local starburst galaxies. Sources are compiled in Table A.1 in Becker et al. (2009). 

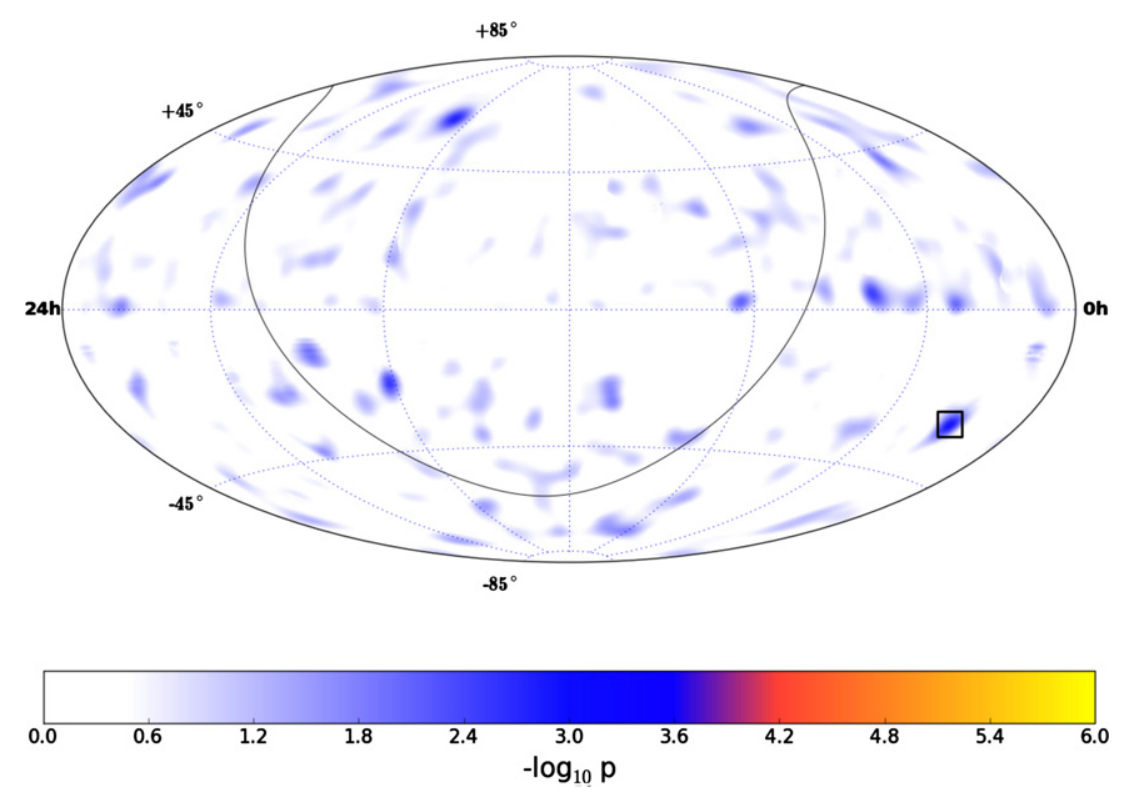

Figure 8. Same as Figure 6, but for sources of $3^{\circ}$ extension.

(A color version of this figure is available in the online journal.)
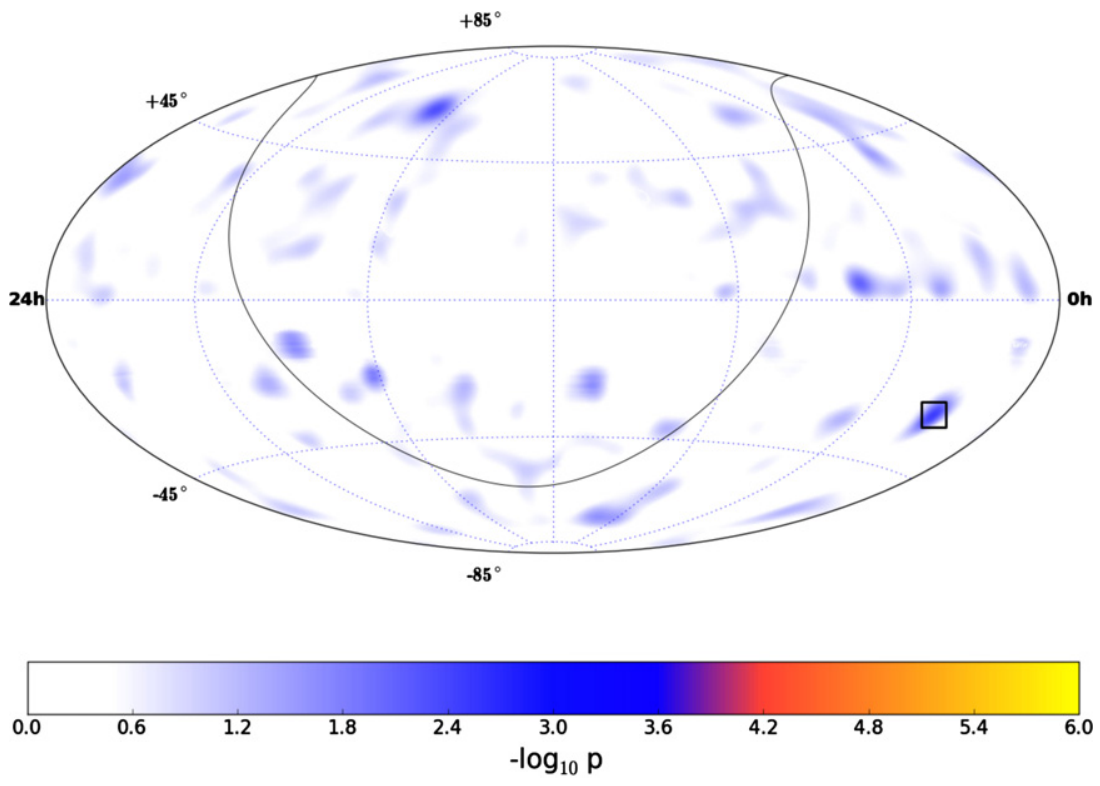

Figure 9. Same as Figure 6, but for sources of $4^{\circ}$ extension.

(A color version of this figure is available in the online journal.)

Five nearby clusters of galaxies. This search tests four models assuming different CR spatial distribution within the source (Murase et al. 2008).

Ten SNRs associated with molecular clouds. This search is now updated to include more sources in the southern sky owing to our increased sensitivity in the southern sky due to new background rejection techniques. From the exhaustive online catalog SNRCat (Ferrand \& Safi-Harb 2012), we select sources with confirmed molecular clouds associations. In order to keep the most promising neutrino emitters within the catalog, only sources that have been observed in the $\mathrm{TeV}$ or are younger than 10,000 yr (potentially in the Sedov blast wave phase (Sedov 1946) of expansion) are considered. The catalog contains four SNRs associated with molecular clouds in the northern sky (Abdo et al. 2007, 2009a, 2009b, 2010; Ackermann et al.
2013; Fiasson et al. 2009) that were previously considered in Aartsen et al. (2013c), and six newly introduced sources from SNRCat in the southern sky. These six sources are Sgr A East, Kes 75, 3C391, RX J1713.7-3946, CTB 37A, and 1FGL J1717.9-3729.

Two hundred and thirty-three galaxies with super-massive black holes. A sample of AGNs within the GZK (Greisen-Zatsespin-Kuzmin; Greisen 1966) radius as cataloged by Caramete \& Biermann (2010) keeping only sources more massive than $5 \times 10^{8}$ solar masses.

New searches. These are new searches introduced with the inclusion of the first year of data from the completed 86 string detector.

Ten Galactic pulsar wind nebulae. Pulsar wind nebulae (PWNs) are potential emitters of neutrinos (Bednarek 

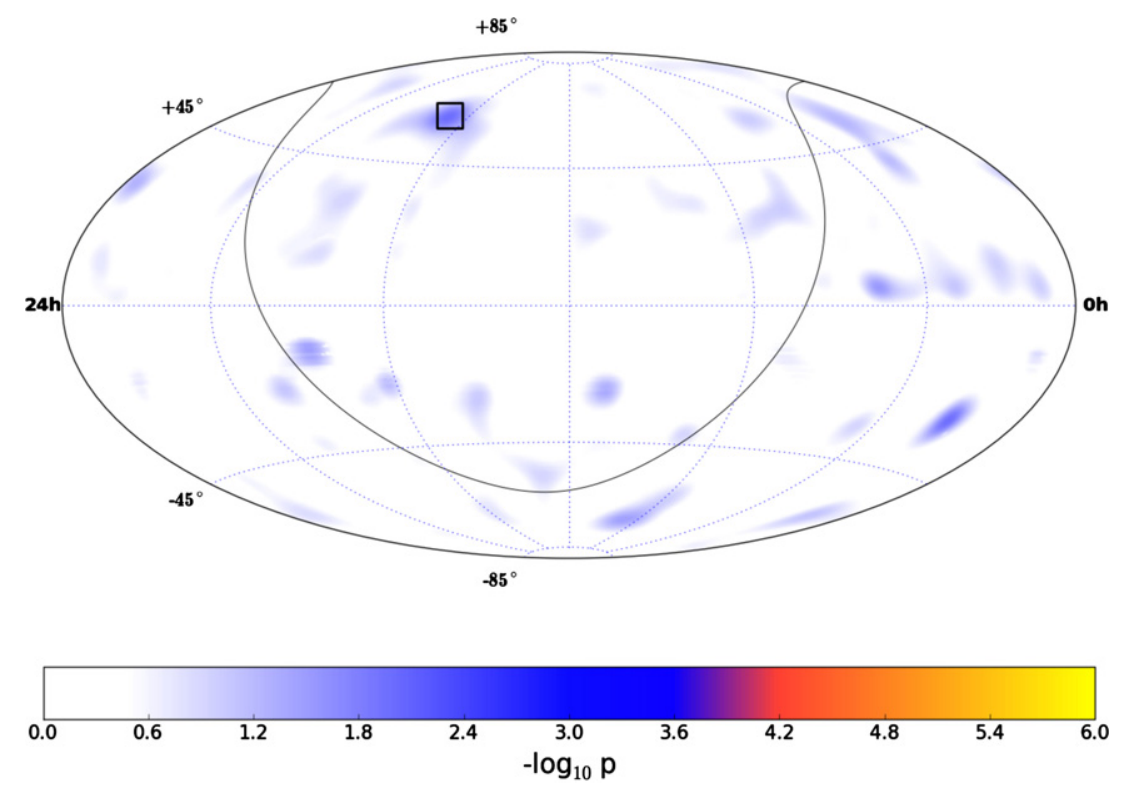

Figure 10. Same as Figure 6, but for sources of $5^{\circ}$ extension.

(A color version of this figure is available in the online journal.)

2003). We carry out a stacked search for neutrinos coming from known PWNs within the Galaxy. From the confirmed PWNs in SNRCat (Ferrand \& Safi-Harb 2012), we look at sources that are younger than $10,000 \mathrm{yr}$ as only younger PWNs are efficient accelerators (Bednarek 2003). We leave out sources that are already considered by the search for SNRs associated with molecular clouds. These criteria are fulfilled by three sources in the northern sky, namely, the Crab Nebula, DA 530, and G054.1+00.3, and seven sources in the southern sky including the Pencil Nebula, W33, and MSH 11-54. These sources are weighted in likelihood by the inverse of their median age as provided by SNRCat (Ferrand \& Safi-Harb 2012) to account for the higher fluxes expected from the youngest PWNs (Bednarek 2003).

Thirty Galactic SNRs. Galactic SNRs (Ferrand \& Safi-Harb 2012) that neither have confirmed molecular cloud associations nor are PWNs are considered in this stacking search. As in the searches for PWNs and SNRs with molecular cloud associations, a cut on the SNR age is applied and only those younger than 10,000 yr are selected (Castro et al. 2011). This requirement is met by 30 sources in total, where 20 are located in the southern sky and 10 in the north. The inverse of the median age as provided by SNRCat (Ferrand \& Safi-Harb 2012) is used as the weight for each source in likelihood in order to account for the fact that we expect the highest fluxes to come from the youngest SNRs. Remnants of recent prominent supernovae such as Casseopeia A and Tycho are considered within this search.

Blazars catalogs. Three blazar catalogs were composed from the Fermi LAT Second AGN catalog (Ackermann et al. 2011) to allow for optimized analyses of the corresponding object classes. The first catalog contains flat spectrum radio quasars (FSRQs), which, as suggested by their broad line regions, are thought to provide efficient photomeson production (Atoyan \& Dermer 2001) in dense soft photon targets. The second set is formed by low-frequency peaked (LSP) BL Lac objects that are predicted to show a significant contribution from pion decays to the overall gamma-emission in the synchrotron proton blazar model
(Mücke et al. 2003). Finally, $p-p$ interaction models are covered by a catalog of the BL Lac objects with particularly hard gamma spectra and correspondingly large effective areas for neutrinos in IceCube (Neronov \& Ribordy 2009).

The source selection and weighting for the FSRQ and LSP BL Lac catalogs, assuming prevalence of photohadronic neutrino production, is based on the Fermi LAT gamma-flux. This motivates a weighting that is based on the measured gamma-fluxes but assumes the same spectral index for all sources (hereby denoted by W1).

In proton-proton interaction models, the energy spectrum of the produced neutral secondaries follows the initial CR spectrum down to a threshold below $1 \mathrm{GeV}$. The observation of the gamma spectrum thus allows for a direct prediction of the proton spectrum behavior in the $\mathrm{TeV}$ range, which can be extrapolated to $\mathrm{PeV}$ energies to estimate the neutrino spectrum. Such an approach is not as easily possible for proton-gamma interaction models, as these typically have a lower-energy threshold above TeV energies so that the photon (and neutrino) spectrum below the threshold does not allow for the derivation of the proton spectrum (Neronov \& Ribordy 2009). Hence, the third catalog of hard gamma-spectrum BL Lac objects motivates a selection and weighting based on the number of detectable neutrinos derived from the spectral shape measured by Fermi LAT (hereby denoted by W2).

Due to the variety of blazar models and the large model uncertainties, both weighting schemes are applied to all three catalogs. Sources with negligible weights in both weighting schemes are discarded, resulting in 33 FSRQs, 27 LSP BL Lac objects, and 37 hard gamma-spectrum BL Lac objects.

This stacked search for blazars uses a reprocessed data set of the 79 string configuration that incorporates the new reconstruction methods presented in this work for IC-86, which were not yet available at the time of the previous analyses. 


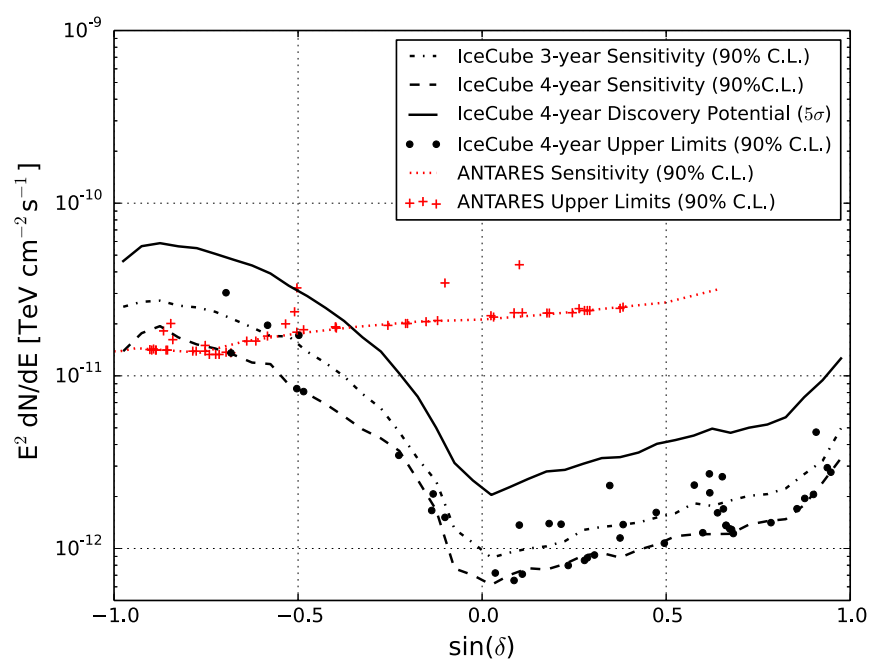

Figure 11. Muon neutrino upper limits with $90 \%$ C.L. evaluated for the 44 sources (dots) for the combined four years of data $(40,59,79$, and 86 string detector configurations). The solid black line is the flux required for $5 \sigma$ discovery of a point source emitting an $E^{-2}$ flux at different declinations, while the dashed line is the median upper limit or sensitivity also for a $90 \%$ C.L. The sensitivity of this search represents a $40 \%-50 \%$ improvement compared to previous IceCube results (dash-dotted line; Aartsen et al. 2013c). The ANTARES sensitivities and upper limits are also shown (Adrián-Martínez et al. 2014). For sources in the southern hemisphere, ANTARES constrains neutrino fluxes at lower energies than this work.

(A color version of this figure is available in the online journal.)

\section{RESULTS AND IMPLICATIONS}

In this section, we summarize all the results from the different searches and their implication on astrophysical models of neutrino emission. While no significant excess has been found in any of the searches and all results are consistent with the background-only hypothesis, this has allowed us to set upper limits that exclude some of the models.

\subsection{All-sky Searches}

\subsubsection{All-sky Point Source Scan}

Figure 5 shows the result of the all-sky scan for point sources in terms of significance at each location in the sky given in equatorial coordinates. The most significant deviation in the northern sky has a pre-trial $p$-value of $4.81 \times 10^{-6}$ and is located at $29.25 \mathrm{R}$.A. and $10.55 \mathrm{decl}$. At this location, the best-fit values of the number of source events, $\hat{n}_{s}$, and signal spectral index, $\hat{\gamma}$, are 43.0 and 2.88 , respectively. In the southern sky, the most significant deviation has a pre-trial $p$-value of $6.81 \times 10^{-6}$ and is located at 347.95 R.A. and -57.75 decl. Here, the best-fit values of $\hat{n}_{s}$ and $\hat{\gamma}$ are 13.0 and 3.95, respectively. After accounting for the trial factor associated with scanning the sky for the most significant spots, the post-trial $p$-values are 0.23 for the spot located in the northern sky and 0.44 for the spot located in the southern sky.

\subsubsection{All-sky Scans for Extended Sources}

Table 2 summarizes the most significant hotspots in the sky from the scans for sources of various extensions. All observations were compatible with the background hypothesis. Figures $6-10$ show the corresponding skymaps for $1^{\circ}, 2^{\circ}, 3^{\circ}$, $4^{\circ}$, and $5^{\circ}$ extension, respectively.

Since filtering streams, reconstructions, and detector configurations evolved with time, we also examined each of the four years of data independently as an a posteriori cross-check. The

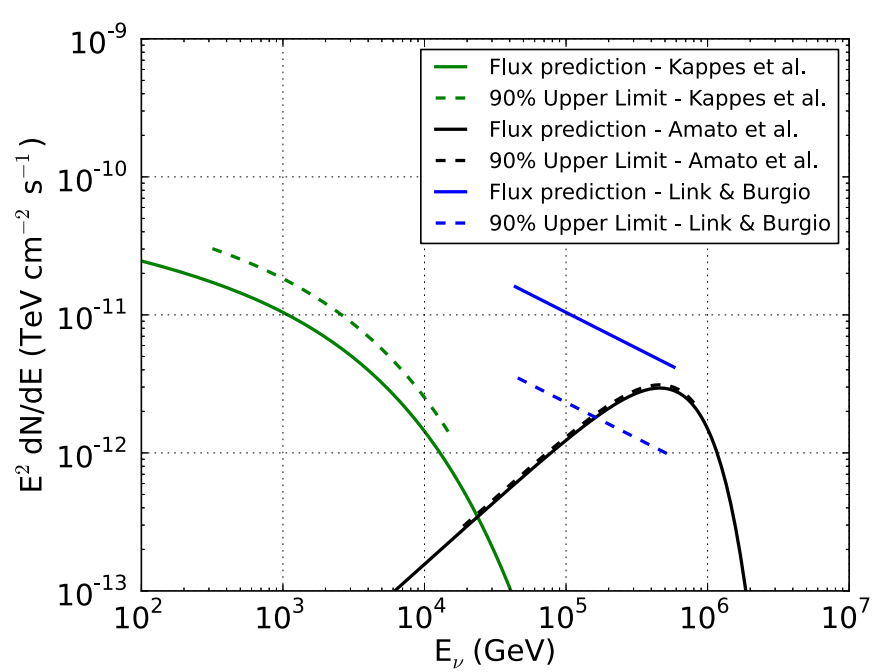

Figure 12. Flux predictions (solid) for three models of neutrino emission from the Crab Nebula, with their associated $90 \%$ C.L. upper limits (dashed) for an energy range containing $90 \%$ of the signal. Both the model from Amato et al. (2003) and the most optimistic model from Link \& Burgio $(2005,2006)$ are now excluded at $90 \%$ C.L. For the gamma-ray-based model from Kappes et al. (2007), the upper limit is still a factor of 1.75 above the prediction.

(A color version of this figure is available in the online journal.)

largest fluctuation was observed for the $1^{\circ}$ extension hypothesis in data from the 79 string configuration at 266.75 R.A. and 13.25 decl., where $0.35 \%$ of scrambled maps in that year resulted in a fluctuation more significant than the one observed. Since we scanned over five different extensions for every year, the corresponding trial-corrected $p$-value is $7.2 \%$, well compatible with a background fluctuation. The hot-spot seems to be driven by a single well-reconstructed very high-energy event which, when folded with the wider source template, overlaps with some nearby lower-energy ones. From calibration using the shadow of the Moon (Aartsen et al. 2013d), there is no evidence for a systematic error in IceCube's point-spread function that could lead to the observed spread for events originating from a point-like source. The region is not significant in any of the other years of data.

\subsection{List of 44 Candidate Sources}

The search for neutrino emission from an a priori list of 44 candidate sources produced the results shown in Tables 3 and 4 . In the northern sky, 1ES 0229+200 has the strongest upward fluctuation. The pre-trial $p$-value of such a fluctuation is 0.053 , but after considering the random chance of observing a fluctuation as strong or stronger than this in any of the sources, the post-trial $p$-value is 0.61 . In the southern sky, PKS 0537-441 has the strongest upward fluctuation, with a pre-trials $p$-value of 0.083 and a post-trials $p$-value of 0.33 . Upper limits on the $E^{-2}$ muon neutrino flux for $90 \%$ confidence level (C.L.) from each source are listed in the table, and are shown along with the analysis sensitivity in Figure 11.

While many baseline models for CR acceleration and highenergy neutrino production predict $E^{-2}$ neutrino spectra, individual sources with unique conditions can produce significantly different spectra. Models for any source in the sky can be tested with the analysis method used in this work, and a number of individual sources were previously considered in Aartsen et al. (2013c). Here, we update the $90 \%$ C.L. upper limits on three models of neutrino emission from the Crab Nebula (Figure 12) as well as three Galactic SNRs (Figure 13). 
Table 3

Results for Galactic Objects on the A Priori Search List

\begin{tabular}{lcrrrrrrr}
\hline \hline Category & Source & R.A. $\left({ }^{\circ}\right)$ & Decl. $\left({ }^{\circ}\right)$ & $p$-value & $\hat{n}_{S}$ & $\hat{\gamma}$ & $B_{1^{\circ}}$ & $\Phi_{v_{\mu}+\bar{\nu}_{\mu}}^{90 \%}$ \\
\hline SNR & Tycho & 6.36 & 64.18 & $\ldots$ & 0.0 & $\ldots$ & 17.8 & 2.06 \\
& Cas A & 350.85 & 58.81 & $\ldots$ & 0.0 & $\ldots$ & 17.8 & 1.70 \\
& IC443 & 94.18 & 22.53 & 0.35 & 4.6 & 3.9 & 27.8 & 1.38 \\
\hline HMXB & LSI +63 303 & 40.13 & 61.23 & $\ldots$ & 0.0 & $\ldots$ & 17.8 & 1.95 \\
/mqso & Cyg X-3 & 308.11 & 40.96 & 0.42 & 3.7 & 3.9 & 21.5 & 1.70 \\
& Cyg X-1 & 299.59 & 35.20 & 0.18 & 8.9 & 3.9 & 23.4 & 2.33 \\
& HESS J0632+057 & 98.25 & 5.80 & 0.14 & 13.4 & 3.4 & 37.0 & 1.37 \\
& SS433 & 287.96 & 4.98 & $\ldots$ & 0.0 & $\ldots$ & 37.6 & 0.65 \\
\hline Star formation region & Cyg OB2 & 308.08 & 41.51 & $\ldots$ & 0.0 & $\ldots$ & 21.0 & 1.36 \\
\hline Pulsar/ & MGRO J2019+37 & 305.22 & 36.83 & $\ldots$ & 0.0 & $\ldots$ & 23.1 & 1.23 \\
PWN & Crab Nebula & 83.63 & 22.01 & 0.44 & 4.4 & 3.9 & 27.8 & 1.15 \\
& Geminga & 98.48 & 17.77 & $\ldots$ & 0.0 & $\ldots$ & 30.7 & 0.92 \\
\hline Galactic center & Sgr A* & 266.42 & -29.01 & $\ldots$ & 0.0 & $\ldots$ & 36.6 & 8.11 \\
Not identified & MGRO J1908+06 & 286.98 & 6.27 & $\ldots$ & 0.0 & $\ldots$ & 36.4 & 0.71 \\
\hline
\end{tabular}

Notes. Sources are grouped according to their classification as high-mass X-ray binaries or micro-quasars (HMXB/ mqso), SNRs, pulsar wind nebulae (PWNs), star formation regions, and unidentified sources. The $p$-value is the pre-trial probability of compatibility with the background-only hypothesis. The $\hat{n}_{S}$ and $\hat{\gamma}$ columns give the best-fit number of signal events and spectral index of a power-law spectrum. When $\hat{n}_{S}=0$, no $p$-value or $\hat{\gamma}$ are reported. The eighth column gives the number of background events in a circle of $1^{\circ}$ around the search coordinates. The last column shows the upper limits based on the classical approach (Neyman 1937) for an $E^{-2}$ flux normalization of $v_{\mu}+\bar{v}_{\mu}$ flux in units of $10^{-12} \mathrm{TeV}^{-1} \mathrm{~cm}^{-2} \mathrm{~s}^{-1}$.

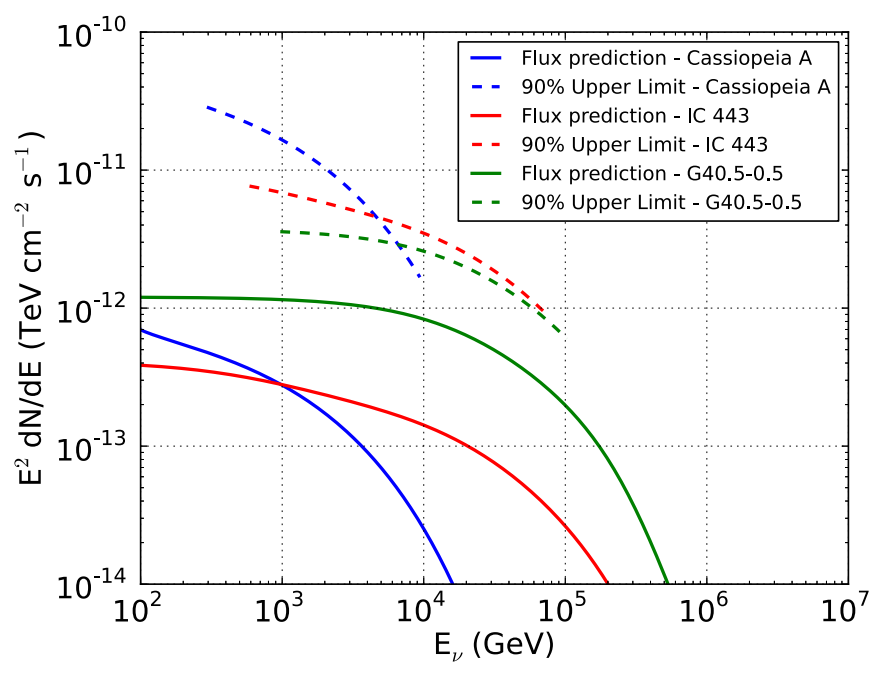

Figure 13. Flux predictions (solid) and upper limits (dashed) for three Galactic supernova remnants. The neutrino models, based of fitted gamma-ray observations, are from (Mandelartz \& Tjus 2013). For the source with the highest predicted flux, G40.5-0.5, the upper limit is a factor of three above the model. (A color version of this figure is available in the online journal.)

\subsection{Stacking Searches}

The results of all stacking searches are compatible with the background-only hypothesis and are summarized in Table 5. The most significant deviation from the background-only hypothesis was observed in the stacked search for neutrino emission from the six Milagro TeV gamma-ray sources, with a $p$-value of 0.02 . The fitted spectral index of 3.95, however, suggests that only low-energy events contribute toward the observation and the observed significance is from spatial clustering only. While Halzen et al. (2008) predicts a flux of much higher energy neutrinos from these sources, the assumptions made about the gamma-ray spectra of the sources in Halzen et al. (2008)

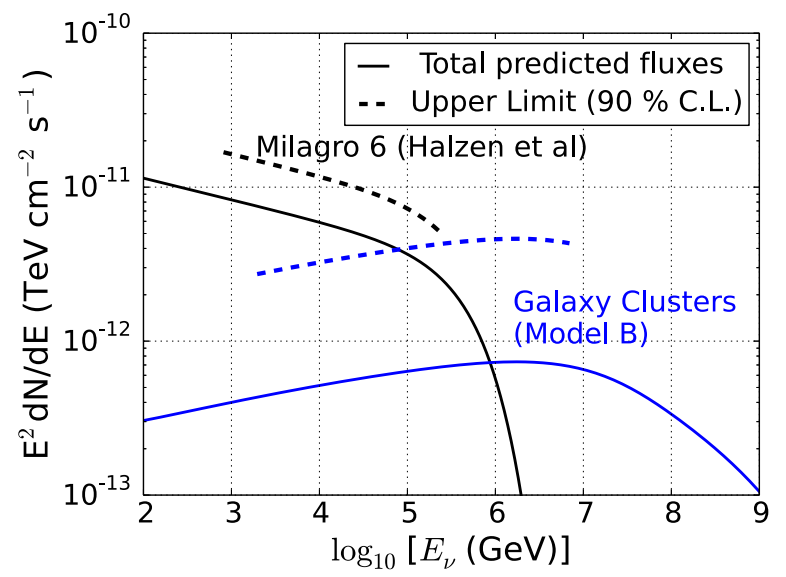

Figure 14. IceCube 90\% C.L. upper limits to the models of Halzen et al. (2008) and Murase et al. (2008).

(A color version of this figure is available in the online journal.)

have later proved to be too optimistic (Abdo et al. 2012). Subsequently, the authors have updated the models (GonzalezGarcia et al. 2014). Figure 14 shows the IceCube upper limits to the model of Halzen et al. (2008). In Figure 14, we also compare limits on neutrino fluxes from galaxy clusters to the model from Murase et al. (2008).

\subsection{Systematic Uncertainties}

In all analyses described here, the background is estimated by scrambling the detector data in right ascension and is independent of theoretical uncertainties on fluxes of atmospheric neutrino and muons as well as uncertainties in the simulation of the detector. The $p$-values are therefore robust against most sources of systematic error. Upper limits and analysis sensitivities, however, are calculated by simulating the detector response to neutrinos. Detector uncertainties including the optical properties of the ice and the absolute efficiency of the 
Table 4

Results for Extragalactic Objects on the A Priori Search List

\begin{tabular}{|c|c|c|c|c|c|c|c|c|}
\hline Category & Source & R.A. $\left({ }^{\circ}\right)$ & Decl. $\left({ }^{\circ}\right)$ & $p$-value & $\hat{n}_{S}$ & $\hat{\gamma}$ & $B_{1^{\circ}}$ & $\Phi_{v_{\mu}+\bar{v}_{\mu}}^{90 \%}$ \\
\hline \multirow[t]{13}{*}{ BL Lac object } & S5 0716+71 & 110.47 & 71.34 & & 0.0 & $\ldots$ & 16.5 & 2.77 \\
\hline & 1ES $1959+650$ & 300.00 & 65.15 & 0.083 & 9.8 & 3.2 & 17.7 & 4.72 \\
\hline & 1ES $2344+514$ & 356.77 & 51.70 & $\ldots$ & 0.0 & $\ldots$ & 19.1 & 1.41 \\
\hline & 3C66A & 35.67 & 43.04 & $\ldots$ & 0.0 & $\ldots$ & 20.5 & 1.220 \\
\hline & H $1426+428$ & 217.14 & 42.67 & $\ldots$ & 0.0 & $\ldots$ & 20.8 & 1.29 \\
\hline & BL Lac & 330.68 & 42.28 & $\ldots$ & 0.0 & $\ldots$ & 20.8 & 1.30 \\
\hline & Mrk 501 & 253.47 & 39.76 & 0.45 & 3.2 & 3.7 & 22.1 & 1.61 \\
\hline & Mrk 421 & 166.11 & 38.21 & 0.26 & 3.8 & 1.9 & 22.4 & 2.10 \\
\hline & W Comae & 185.38 & 28.23 & 0.34 & 1.4 & 1.6 & 25.9 & 1.62 \\
\hline & 1ES $0229+200$ & 38.20 & 20.29 & $0.053^{\mathrm{a}}$ & 16.0 & 3.7 & 28.6 & 2.32 \\
\hline & PKS 0235+164 & 39.66 & 16.62 & $\ldots$ & 0.0 & $\ldots$ & 31.4 & 0.88 \\
\hline & PKS 2155-304 & 329.72 & -30.23 & $\ldots$ & 0.0 & $\ldots$ & 37.0 & 8.43 \\
\hline & PKS 0537-441 & 84.71 & -44.09 & $0.083^{b}$ & 6.3 & 3.9 & 35.2 & 30.03 \\
\hline \multirow[t]{11}{*}{ FSRQ } & $4 C 38.41$ & 248.81 & 38.13 & 0.12 & 10.6 & 2.8 & 22.4 & 2.71 \\
\hline & 3C 454.3 & 343.49 & 16.15 & $\ldots$ & 0.0 & $\ldots$ & 31.4 & 0.85 \\
\hline & PKS $0528+134$ & 82.73 & 13.53 & $\ldots$ & 0.0 & $\ldots$ & 32.3 & 0.80 \\
\hline & PKS $1502+106$ & 226.10 & 10.49 & 0.21 & 6.1 & 2.3 & 33.2 & 1.39 \\
\hline & $3 \mathrm{C} 273$ & 187.28 & 2.05 & 0.45 & 3.2 & 2.6 & 38.9 & 0.72 \\
\hline & $3 \mathrm{C} 279$ & 194.05 & -5.79 & & 0.0 & $\ldots$ & 33.5 & 1.51 \\
\hline & QSO $2022-077$ & 306.42 & -7.64 & 0.45 & 1.3 & 2.0 & 34.1 & 2.07 \\
\hline & PKS 1406-076 & 212.24 & -7.87 & $\ldots$ & 0.0 & $\ldots$ & 34.1 & 1.66 \\
\hline & QSO $1730-130$ & 263.26 & -13.08 & $\ldots$ & 0.0 & $\ldots$ & 37.1 & 3.46 \\
\hline & PKS 1622-297 & 246.53 & -29.86 & 0.13 & 6.2 & 2.7 & 36.6 & 17.17 \\
\hline & PKS 1454-354 & 224.36 & -35.65 & 0.2 & 5.4 & 3.9 & 35.6 & 19.64 \\
\hline \multirow{6}{*}{$\begin{array}{l}\text { Starburst } \\
\text { radio } \\
\text { galaxies }\end{array}$} & M82 & 148.97 & 69.68 & & 0.0 & $\ldots$ & 16.3 & 2.94 \\
\hline & NGC 1275 & 49.95 & 41.51 & $\ldots$ & 0.0 & $\ldots$ & 21.0 & 1.36 \\
\hline & Cyg A & 299.87 & 40.73 & 0.18 & 1.8 & 1.5 & 21.5 & 2.60 \\
\hline & $3 \mathrm{C} 123.0$ & 69.27 & 29.67 & $\ldots$ & 0.0 & $\ldots$ & 25.7 & 1.07 \\
\hline & M87 & 187.71 & 12.39 & 0.26 & 8.8 & 3.9 & 32.4 & 1.38 \\
\hline & Cen A & 201.37 & -43.02 & $\ldots$ & 0.0 & $\ldots$ & 35.5 & 13.57 \\
\hline
\end{tabular}

Notes. Sources are grouped according to their classification as BL Lac objects, radio galaxies, FSRQs, and starburst galaxies. The $p$-value is the pre-trial probability of compatibility with the background-only hypothesis. The $\hat{n}_{S}$ and $\hat{\gamma}$ columns give the best-fit number of signal events and spectral index of a power-law spectrum. When $\hat{n}_{S}=0$, no $p$-value or $\hat{\gamma}$ are reported. The eighth column gives the number of background events in a circle of $1^{\circ}$ around the search coordinates. The last column shows the upper limits based on the classical approach (Neyman 1937) for an $E^{-2}$ flux normalization of $v_{\mu}+\bar{v}_{\mu}$ flux in units of $10^{-12} \mathrm{TeV}^{-1} \mathrm{~cm}^{-2} \mathrm{~s}^{-1}$.

${ }^{a}$ Most significant $p$-value in the northern sky among all Galactic and extragalactic objects on the a priori search list.

${ }^{\mathrm{b}}$ Most significant $p$-value in the southern sky among all Galactic and extragalactic objects on the a priori search list.

Table 5

Results of the Stacked Searches for Emission from Source Catalogs

\begin{tabular}{lccccc}
\hline \hline Catalog & & $\hat{n}_{S}$ & $\hat{\gamma}$ & $p$-value & $\Phi_{v_{\mu}+\bar{v}_{\mu}}^{90 \%}$ \\
\hline Milagro 6 & & 51.4 & 3.95 & 0.02 & $1.98 \times$ M.F. (Halzen et al. 2008) \\
Galaxy clusters & Model A & 1.4 & 4.07 & 0.50 & $3.89 \times$ M.F. (Murase et al. 2008) \\
& Model B & 12.6 & 3.95 & 0.48 & $6.30 \times$ M.F. (Murase et al. 2008) \\
& Central AGN & 0.0 & $\ldots$ & $\ldots$ & $1.59 \times$ M.F. (Murase et al. 2008) \\
& Isobaric & 0.0 & $\ldots$ & $\ldots$ & $4.79 \times$ M.F. (Murase et al. 2008) \\
Starburst galaxies & & 0.0 & $\ldots$ & $\ldots$ & $7.93 \times 10^{-12} \times E^{2.0}$ \\
MC associated SNRs & & 0.0 & $\ldots$ & $\ldots$ & $1.60 \times 10^{-9} \times E^{2.7}$ \\
Supermassive black holes & & 17.1 & 3.95 & 0.43 & $6.88 \times 10^{-12} \times E^{2.0}$ \\
Young SNRs & & 0.0 & $\ldots$ & $\ldots$ & $4.83 \times 10^{-12} \times E^{2.0}$ \\
Young PWNs & & 0.0 & $\ldots$ & $\ldots$ & $3.12 \times 10^{-12} \times E^{2.0}$ \\
FSRQs & W1 & 9.8 & 2.45 & 0.31 & $3.46 \times 10^{-12} \times E^{2.0}$ \\
& W2 & 15.4 & 2.75 & 0.19 & $34.3 \times$ M.F. \\
LSP BL Lac objects & W1 & 11.9 & 3.25 & 0.38 & $5.24 \times 10^{-12} \times E^{2.0}$ \\
& W2 & 21.8 & 3.59 & 0.10 & $13.5 \times$ M.F. \\
Hard BL Lac objects & W1 & 0 & $\ldots$ & $\ldots$ & $3.73 \times 10^{-12} \times E^{2.0}$ \\
& W2 & 17.5 & 3.95 & 0.29 & $0.284 \times$ M.F. \\
\hline
\end{tabular}

Notes. M.F. stands for the model flux as described in the references motivating the analyses. $\Phi_{v_{\mu}+\bar{v}_{\mu}}^{90 \%}$ is the $90 \%$ confidence level upper limit on the combined flux of $v_{\mu}$ and $\bar{v}_{\mu}$ from the catalogs. The $E^{2.0}$ limits are in units of $\mathrm{TeV}^{1} \mathrm{~cm}^{-2} \mathrm{~s}^{-1}$. 


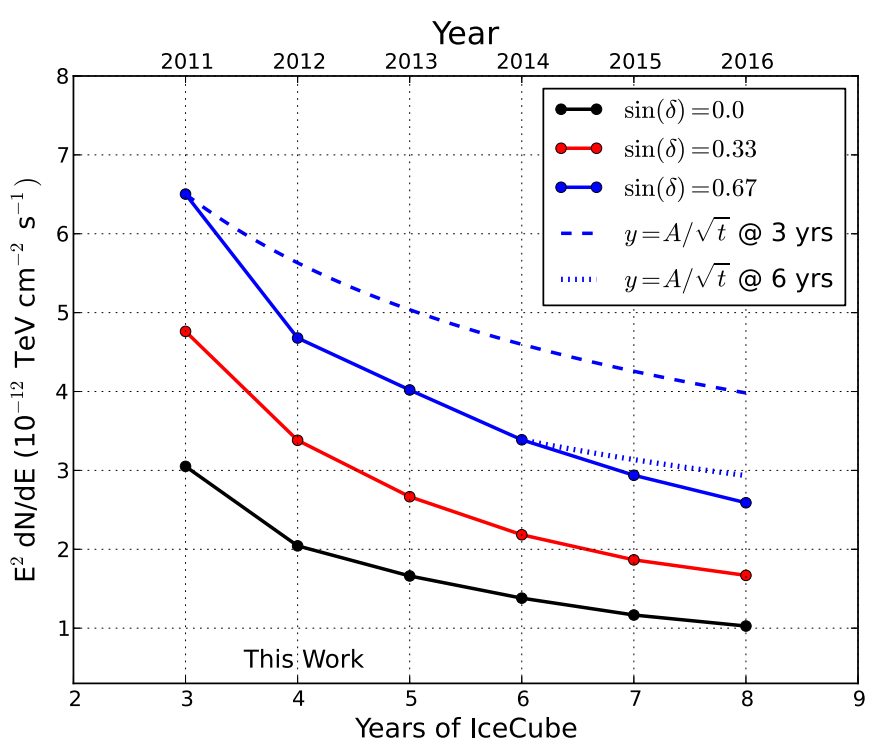

Figure 15. Predicted $E^{-2}$ discovery potential as a function of years of running time of the IceCube Observatory for three different declinations (solid lines). Due to the relatively low background rate in this analysis, the discovery potential will continue to improve faster than the square-root of time limit (dashed and dotted lines).

(A color version of this figure is available in the online journal.)

optical modules can affect the reported sensitivities and upper limits.

After a detailed discussion of all relevant systematic uncertainties, Aartsen et al. (2013c) concludes that the level of uncertainty in the analysis using three years of data is about $18 \%$. Since $65 \%$ of the data used here is the same as in Aartsen et al. (2013c) and the techniques for the new event selection and analyses are similar, the systematic uncertainty on the four year sample is about the same. However, the added year of data utilizes a new muon track reconstruction, which is more sensitive to uncertainties in the optical properties of the ice. We re-evaluate the effect of the ice properties on the analysis for the 2011-2012 data, finding a corresponding systematic uncertainty of $+16 \% /-8 \%$. This is incorporated into the overall systematic uncertainty by averaging it with the ice model effect from the previous years. The resulting overall systematic uncertainty on the quoted sensitivities and upper limits is $21 \%$.

\section{CONCLUSIONS}

No evidence of neutrino emission from point-like or extended sources was found in four years of IceCube data. Searches for emissions from point-like and extended sources anywhere in the sky, from a pre-defined candidate source list, and from stacked source catalogs all returned results consistent with the background-only hypothesis. Ninety percent C.L. upper limits on the muon neutrino fluxes for models from a variety of sources were calculated and compared to predictions. The most optimistic models considered here can be excluded at $90 \%$ C.L. and in other cases limits are a factor of two to four above the predictions. This analysis includes data from the completed IceCube array, taken between 2011 May and 2012 May. IceCube will continue to run in this configuration for the foreseeable future. Future analyses will benefit from this improved integration time and the evolution of the analysis sensitivity as a function of years of data taking is shown in Figure 15. Within a few years, the analyses will surpass the sensitivity necessary to test a wider variety of neutrino point source models. Future developments in background rejection techniques and reconstruction algorithms may lead to improvements faster than predicted in Figure 15.

We acknowledge the support from the following agencies: U.S. National Science Foundation-Office of Polar Programs, U.S. National Science Foundation-Physics Division, University of Wisconsin Alumni Research Foundation, the Grid Laboratory Of Wisconsin (GLOW) grid infrastructure at the University of Wisconsin-Madison, the Open Science Grid (OSG) grid infrastructure; U.S. Department of Energy, and National Energy Research Scientific Computing Center, the Louisiana Optical Network Initiative (LONI) grid computing resources; Natural Sciences and Engineering Research Council of Canada, WestGrid and Compute/Calcul Canada; Swedish Research Council, Swedish Polar Research Secretariat, Swedish National Infrastructure for Computing (SNIC), and Knut and Alice Wallenberg Foundation, Sweden; German Ministry for Education and Research (BMBF), Deutsche Forschungsgemeinschaft (DFG), Helmholtz Alliance for Astroparticle Physics (HAP), Research Department of Plasmas with Complex Interactions (Bochum), Germany; Fund for Scientific Research (FNRS-FWO), FWO Odysseus programme, Flanders Institute to encourage scientific and technological research in industry (IWT), Belgian Federal Science Policy Office (Belspo); University of Oxford, United Kingdom; Marsden Fund, New Zealand; Australian Research Council; Japan Society for Promotion of Science (JSPS); the Swiss National Science Foundation (SNSF), Switzerland; National Research Foundation of Korea (NRF); Danish National Research Foundation, Denmark (DNRF).

\section{REFERENCES}

Aartsen, M. G., Abbasi, R., Abdou, Y., et al. (IceCube Coll.) 2013a, NIMPA, 711,73

Aartsen, M. G., Abbasi, R., Abdou, Y., et al. (IceCube Coll.) 2013b, NIMPA, 736,143

Aartsen, M. G., Abbasi, R, Abdou, Y., et al. (IceCube Coll.) 2013c, ApJ, 779,132

Aartsen, M. G., Abbasi, R., Ackermann, M., et al. (IceCube Coll.) 2014a, JINST, 9, P03009

Aartsen, M. G., Abdou, Y., Ackermann, M., et al. (IceCube Coll.) 2013d, arXiv:1305.6811

Aartsen, M. G., Ackermann, M., Adams, J., et al. (IceCube Coll.) 2014b, PhRvL, 113,101101

Aartsen, M. G., et al. (IceCube Coll.) 2013e, Sci, 342, 1242856

Abbasi, R., Abdou, Y., Abu-Zayyad, T., et al. (IceCube Coll.) 2010, NIMPA, 618,139

Abbasi, R., Abdou, Y., Abu-Zayyad, T., et al. (IceCube Coll.) 2011, ApJ, 732,18

Abbasi, R., Ackermann, M., Adams, J., et al. (IceCube Coll.) 2009, NIMPA, 601,294

Abdo, A. A., Abeysekara, U., Allen, B. T., et al. 2012, ApJ, 753, 159

Abdo, A. A., Ackermann, M., Ajello, M., et al. (Fermi Coll.) 2009a, ApJL, 706, L1

Abdo, A. A., Ackermann, M., Ajello, M., et al. (Fermi Coll.) 2010, Sci, 327,1103

Abdo, A. A., Allen, B., Berley, D., et al. 2007, ApJL, 664, L91

Abdo, A. A., Allen, B. T., Aune, T., et al. (Milagro Coll.) 2009b, ApJL, 700, L127

Achterberg, A., Ackermann, M., Adams, J., et al. (IceCube Coll.) 2006, APh, 26, 155

Ackermann, M., Ajello, M., Allafort, A., et al. 2011, ApJ, 743, 171

Ackermann, M., Ajello, M., Allafort, A., et al. (Fermi Coll.) 2013, Sci, 339,807

Adrián-Martínez, S., Albert, A., André, M., et al. (Antares Coll.) 2014, ApJL, 786, L5

Ahrens, J., Bai, X., Bay, R., et al. 2004, NIMPA, 524, 169

Alvarez-Muñiz, J., \& Halzen, F. 2002, ApJL, 576, L33

Amato, E., Guetta, D., \& Blasi, P. 2003, A\&A, 402, 827

Anchordoqui, L. A., Barger, V., Cholis, I., et al. 2014, JHEAp, 1, 1 
Anchordoqui, L. A., \& Montaruli, T. 2010, ARNPS, 60, 129

Atoyan, A., \& Dermer, C. D. 2001, PhRvL, 87, 221102

Becker, J. 2008, PhR, 458, 173

Becker, J. K., Biermann, P. L., Dreyer, J., \& Kneiske, T. M. 2009, arXiv:0901.1775

Bednarek, W. 2003, A\&A, 407, 1

Braun, J., Baker, M., Dumm, J., et al. 2010, APh, 33, 175

Caramete, L., \& Biermann, P. L. 2010, A\&A, 521, A55

Carrigan, S., Brun, F., Chaves, R. C. G., et al. (HESS Coll.) 2013, in Proc of the 48th Rencontres de Moriond, La Thuile, Italy, ed. E. Auge \& J.

Dumarchez (available online at http://moriond.in2p3.fr/Proceedings/2013/ Moriond_QCD_2013.pdf)

Castro, D., Slane, P., Patnaude, D. J., \& Ellison, D. C. 2011, ApJ, 734, 85

Cavasinni, V., Grasso, D., \& Maccione, L. 2006, APh, 26, 41

De Marco, D., Blasi, P., Hansen, P., \& Stanev, T. 2006, PhRvD, 73, 043004

Essey, W., Kalashev, O. E., Kusenko, A., \& Beacom, J. F. 2010, PhRvL, 104, 141102

Ferrand, G., \& Safi-Harb, S. 2012, AdSpR, 49, 1313

Fiasson, A., Marandon, V., Chaves, R. C. G., \& Tibolla, O. (HESS Coll.) 2009, in Proceedings of the 31st Int. Cosmic Ray Conf., Lodz, Poland, Discovery of a VHE Gamma-Ray Source in the W51 Region, ed. J. Szabelski \& M. Giller (Lodz: Univ. of Lodz)

Gonzalez-Garcia, M. C., Halzen, F., \& Niro, V. 2014, APh, 57, 39

Greisen, K. 1966, PhRvL, 16, 748

Guetta, D., Hooper, D., Alvarez-MuñIz, J., Halzen, F., \& Reuveni, E. 2004, $\mathrm{APh}, 20,429$

Halzen, F., \& Hooper, D. 2002, RPPh, 65, 1025

Halzen, F., Kappes, A., \& O’Murchadha, A. 2008, PhRvD, 78, 063004

Honda, M., Kajita, T., Kasahara, K., Midorikawa, S., \& Sanuki, T. 2007, PhRvD, 75, 043006

Kalashev, O. E., Kusenko, A., \& Essey, W. 2013, PhRvL, 111, 041103
Kappes, A., Hinton, J., Stegmann, C., \& Aharonian, F. A. 2007, ApJ 656,870

Lacki, B. C., Thompson, T. A., Quataert, E., Loeb, A., \& Waxman, E. 2011, ApJ, 734, 107

Learned, J. G., \& Mannheim, K. 2000, ARNPS, 50, 679

Link, B., \& Burgio, F. 2005, PhRvL, 94, 181101

Link, B., \& Burgio, F. 2006, MNRAS, 371, 375

Loeb, A., \& Waxman, E. 2006, JCAP, 05, 003

Mandelartz, M., \& Tjus, J. B. 2013, arXiv:1301.2437

Mészáros, P. 2006, RPPh, 69, 2259

Mücke, A., Protheroe, R. J., Engel, R., Rachen, J. P., \& Stanev, T. 2003, APh, 18,593

Murase, K., Ahlers, M., \& Lacki, B. C. 2013, PhRvD, 88, 121301

Murase, K., \& Beacom, J. F. 2012, JCAP, 02, 028

Murase, K., Inoue, Y., \& Dermer, C. D. 2014, PhRvD, 90, 023007

Murase, K., Inoue, S., \& Nagataki, S. 2008, ApJL, 689, L105

Neronov, A., \& Ribordy, M. 2009, PhRvD, 80, 083008

Neunhöffer, T. 2006, APh, 25, 220

Neyman, J. 1937, RSPTA, 236, 333

Romero, G. E., \& Torres, D. F. 2003, ApJL, 586, L33

Sedov, L. I. 1946, JApMM, 10, 241

Sironi, L., \& Spitkovsky, A. 2011, ApJ, 726, 75

Stecker, F. W., Done, C., Salamon, M. H., \& Sommers, P. 1991, PhRvL, 66, 2697

Tchernin, C., Aguilar, J. A., Neronov, A., \& Montaruli, T. 2013, A\&A, 560, A67

Vissani, F., Aharonian, F., \& Sahakyan, N. 2011, APh, 34, 778

Waxman, E., \& Bahcall, J. 1997, PhRvL, 78, 2292

Waxman, E., \& Bahcall, J. 1999, PhRvD, 59, 023002

Whitehorn, N., van Santen, J., \& Lafebre, S. 2013, CoPhC, 184, 2214

Wolfe, B., Melia, F., Crocker, R. M., \& Volkas, R. R. 2008, ApJ, 687, 193 